\title{
Ensemble-based conditioning of reservoir models to seismic data
}

\author{
Olwijn Leeuwenburgh · Jan Brouwer • Mario Trani
}

Received: 13 December 2009 / Accepted: 1 September 2010 / Published online: 17 September 2010

(C) Springer Science+Business Media B.V. 2010

\begin{abstract}
While 3D seismic has been the basis for geological model building for a long time, time-lapse seismic has primarily been used in a qualitative manner to assist in monitoring reservoir behavior. With the growing acceptance of assisted history matching methods has come an equally rising interest in incorporating $3 \mathrm{D}$ or time-lapse seismic data into the history matching process in a more quantitative manner. The common approach in recent studies has been to invert the seismic data to elastic or to dynamic reservoir properties, typically acoustic impedance or saturation changes. Here we consider the use of both $3 \mathrm{D}$ and time-lapse seismic amplitude data based on a forward modeling approach that does not require any inversion in the traditional sense. Advantages of such an approach may be better estimation and treatment of model and measurement errors, the combination of two inversion steps into one by removing the explicit inversion to state space variables, and more consistent dependence on the validity of assumptions underlying the inversion process. In this paper, we introduce this approach with the use of an assisted history matching method in mind. Two ensemble-based methods, the ensemble Kalman filter and the ensemble randomized maximum likelihood method, are used to investigate
\end{abstract}

O. Leeuwenburgh $(\bowtie) \cdot$ J. Brouwer

TNO, Utrecht, Netherlands

e-mail: olwijn.leeuwenburgh@tno.nl

J. Brouwer

e-mail: jan.brouwer@tno.nl

M. Trani

Delft University of Technology, Delft, Netherlands

e-mail:m.trani@tudelft.nl issues arising from the use of seismic amplitude data, and possible solutions are presented. Experiments with a 3D synthetic reservoir model show that additional information on the distribution of reservoir fluids, and on rock properties such as porosity and permeability, can be extracted from the seismic data. The role for localization and iterative methods are discussed in detail.

Keywords Assisted history matching •

Ensemble Kalman filter • Seismic history matching •

Ensemble randomized maximum likelihood filter

\section{Introduction}

Since the first repeat surveys in the 1980s there has been a growing recognition of the value of (time-lapse) seismic data for reservoir monitoring and modeling. When compaction and repeatability issues can be neglected, time-lapse seismic differences reflect changes in fluid properties, and therefore highlight areas that have either been swept or heated, or rather remain unproduced, valuable information that may not be obtained by other means. If the observed changes are not reproduced by the reservoir model, this information can be used to improve the model by history matching. The large number of data involved in seismic acquisition, however, poses many problems, especially for traditional manual history matching, which is why seismic data has been used primarily in a qualitative way. Recent work on conditioning models to seismic data with so-called 'assisted' history matching methods, suggests that much can potentially be gained by taking a more quantitative approach. 
Stephen et al. [34] and Stephen et al. [35] presented studies of North Sea oil fields where time-lapse seismic was used to constrain reservoir models by applying the Pilot Point Method [7] in combination with the Neighborhood Algorithm [29]. It was concluded that the time-lapse seismic helped to quantify permeability and fault transmissibility and thereby reduce uncertainty in predicted liquid rates and water breakthrough time. Jin et al. [23] applied the Pilot Point Method with an optimization scheme based on Simulated Annealing to estimate porosity in a synthetic 2D model. Dong and Oliver [8] used the adjoint method to update porosity, permeability, pressure and phase saturation in a semisynthetic case, and concluded that a history matched model could be generated with improved prediction skill, and that combination of production and timelapse seismic data helped to resolve the transitions between different property zones.

A history matching method that is gaining growing interest in the reservoir engineering community is the ensemble Kalman filter (EnKF; [12]). An early study which demonstrated the applicability of the method to reservoir history matching based on production data was conducted by Naevdal et al. [26], while Evensen et al. [11] demonstrate an application to a North Sea reservoir model. Two early applications of the EnKF to seismic history matching were presented by Dong et al. [9] and Skjervheim et al. [32]. The seismic attributes that have been mostly used in realistic case studies are acoustic (P-wave) impedance and Poisson ratio, which can be obtained fairly easily by applying a rock physics model to the simulator output, but which require more elaborate inversion schemes to obtain from observed seismic amplitudes. Trani [37] extended the inversion scheme of Landrø [24] with travel-time differences and suggested assimilating the resulting saturation and pressure differences with the EnKF.

Only a few researchers have considered using the seismic waveform data in a more direct manner. Skjervheim and Ruud [33] applied a simple seismic modeling approach to simulate waveform data for a synthetic $2 \mathrm{D}$ reservoir model and used the waveform data together with production data in an EnKFbased updating scheme. Dadashpour et al. [4] and Dadashpour et al. [5] use synthetic time-lapse seismic amplitudes and synthetic time-lapse zero-offset amplitudes plus AVO gradient differences respectively with a 2D model and minimize the difference with simulated data by calculating the sensitivities to unknown grid parameters using finite differences and a Gauss-Newton scheme. Haverl et al. [20] interpreted the GOC depth from single-survey waveform data and assimilated this depth with the EnKF. Most recently, Fahimuddin et al. [13] compared the use of amplitude and impedance data with the EnKF on a sector model based on a North Sea reservoir.

Important consequences of using seismic waveform data directly are that no traditional seismic inversion is required, but that, on the other hand, the seismic response to dynamic changes must be simulated for all model realizations. An advantage of this forward modeling approach is that it is easier to represent uncertainty in the rock physics and seismic models and that it connects more naturally to ensemble-based history matching methods which implicitly represent uncertainty by using a set of model scenarios.

We will adopt this approach in this paper and will apply it to a 3D reservoir model. The forward modeling approach is made feasible by the development of a seismic simulator which incorporates models for rock physics, source wavelet, and several seismic error sources. We will explore the potential of this approach when used in combination with production data for assisted history matching and suggest solutions for practical problems resulting from the use of large amounts of measurements.

Section 2 will start with the introduction of the seismic modeling tool (Section 2.1), and the assisted history matching methods applied here (Section 2.2). In Section 3 several experiments with two synthetic reservoirs will be presented and we will investigate and discuss in detail several alternatives for application of ensemble-based history matching schemes.

\section{Methods}

\subsection{Seismic attribute generation}

A variety of methods may be applied to generate synthetic seismic data. The method most easily implemented is the 1D approach based on a simple convolutional model. In this model it is assumed that the seismic trace may be modeled as the convolution of the seismic source signature and the earth's impulse response,

$y(t)=R(t) * S(t)$,

where $R(t)$ denotes the earth's impulse response, $S(t)$ represents the source wavelet, $y(t)$ represents the seismic trace and $*$ the convolution operator. The earth's impulse response is based on the combined amplitude effects of transmission losses, reflection strength and (spherical) divergence. In our model we address Pwaves only and thus incorporate in our transmission 
and reflection effects only $\mathrm{P}$-wave impedances. The method furthermore assumes a stationary signal (that is, the source wavelet is stationary in time) and does not account for any (frequency dependent) attenuation. As a consequence, the method can only be used to approximate the seismic response in a relatively small time window where attenuation effects can be neglected. Since we are interested only in the seismic response at reservoir depth-and the reservoir thickness is relatively small compared to reservoir depth-the latter assumption may possibly hold, in particular when we deal with fluid filled reservoirs rather than gas filled reservoirs.

Obviously, data simulated using this forward model may diverge significantly from real seismic data. Some of the effects not included in this simple 1D convolution model are (1) additional seismic phases (S-waves, surface-waves) and the conversion between these phases, (2) multiple reflections, (3) "spatial interference" due to limited bandwidth, and (4) acquisition footprint. To account for some of these effects, noise can be added to the synthetic data as follows,

$y(t)=R(t) * S(t)+N(t)$,

where $N(t)$ is the noise term. In our model, the noise is band-limited to the same frequency band as the seismic data and may contain some spatial coherency. This is to account for the processing normally applied to seismic field data that will typically cancel all incoherent noise as well as noise outside the bandwidth of the source wavelet. We furthermore have the option to apply a lateral smoother to $R(t)$ to account for limited spatial bandwidth. Acquisition footprint is currently not taken into account in our seismic simulator, which for timelapse differences implies that ideal repeatability is as- sumed and that none of the acquisition related noise remains.

The earth's impulse response $R(t)$ is derived from the static distribution of density and velocity with depth for the overburden and underburden, and from a rock-physics model that incorporates the pressure and saturation states for the reservoir. These pressure and saturation changes result, for a given rock-matrix and porosity, in changes in velocity and density. In our 1D model velocity changes at reservoir depth affect both travel times and reflectivity, whereas density changes merely affect reflectivity. The rockphysics model is based on the equations introduced by Gassmann [15] and Mindlin [25]. The Mindlin theory calculates effective bulk and shear moduli assuming a dry and dense random pack of identical spherical grains subject to hydrostatic pressure at an initial porosity of 0.36. The Hashin and Shtrikman [19] lower bound estimates the effective moduli at the reservoir porosity, with the assumption that the rock is everywhere isotropic, linear, and elastic. The Gassmann equation [15] predicts the seismic parameters for the saturated rock with the assumption of long wavelengths and low frequencies (corresponding to the seismic range), in a medium where all pores are connected and fluids do not interact with the matrix. An illustration of the resulting changes in seismic attributes as a response to changes in pressure and saturation is provided in Fig. 1.

Figure 2 depicts the sensitivity of the seismic amplitudes (the seismogram) to porosity and saturation perturbations. For the models and type of data used in this study, the amplitudes are rather insensitive to pressure variations. The primary seismic response is associated with the reservoir boundaries with overburden and underburden, resulting in the solid line in Fig. 2a, which is based on an 11-layer reservoir column with uniform porosity and water saturation of 0.2 . Figure $2 \mathrm{a}$
Fig. 1 Rock-physics model for the synthetic seismic model. Changes in elastic properties as a function of changes a saturation, and $\mathbf{b}$ pressure (a)

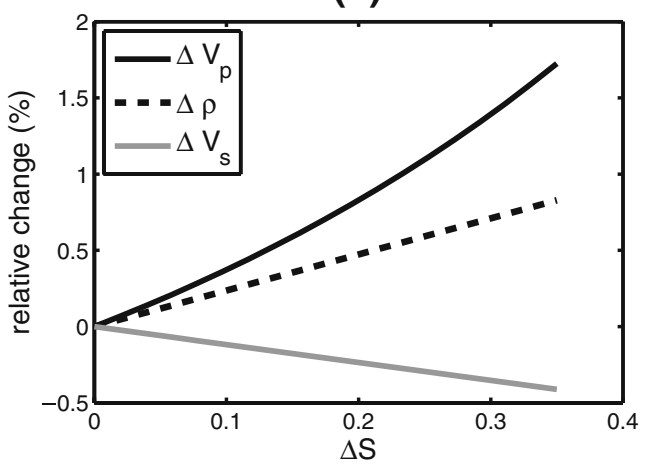

(b)

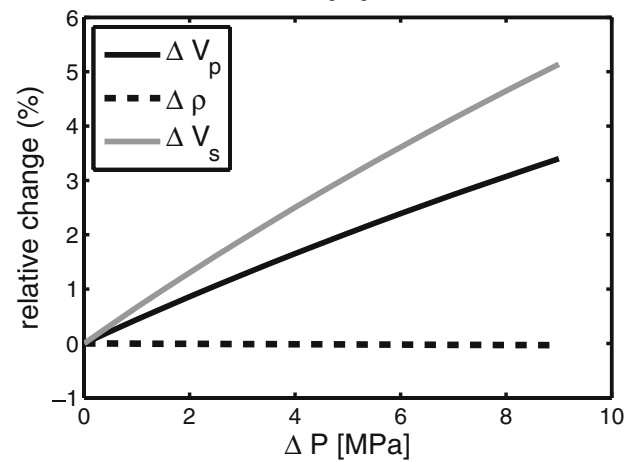


(a)

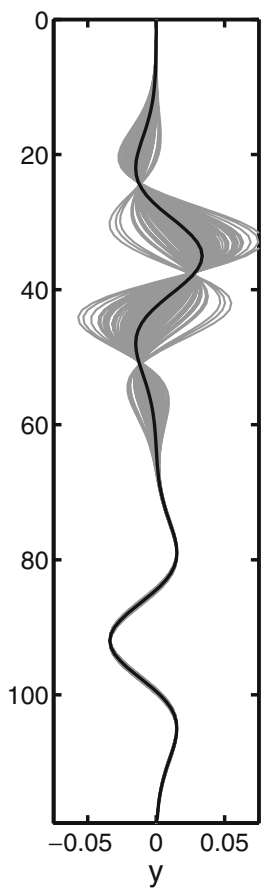

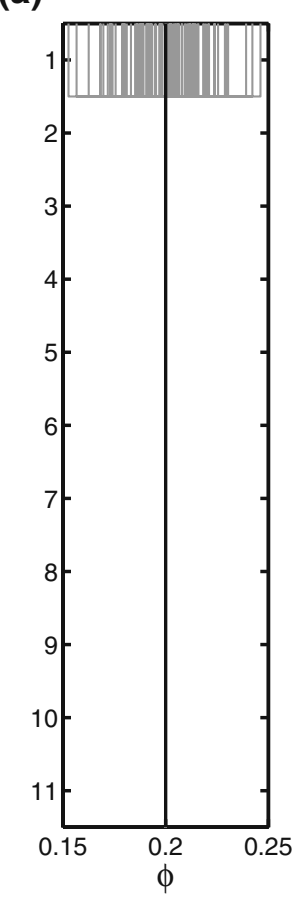

(b)

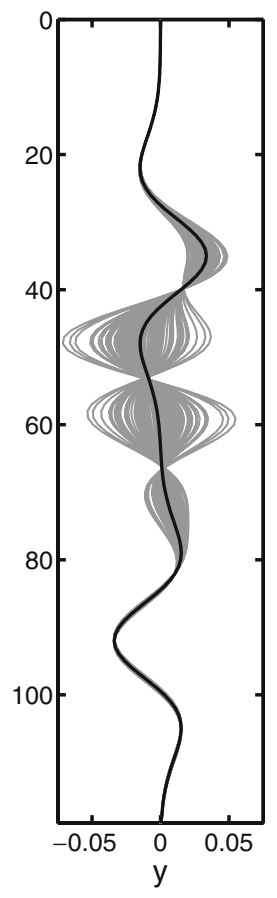

Fig. 2 Seismic amplitude sensitivity to reservoir properties. a Porosity profile and seismic response to porosity perturbation in the top layer. b Porosity profile and seismic response to porosity perturbation in the 4th layer. c Time-lapse saturation

and $\mathrm{b}$ illustrate the instantaneous seismic response to porosity perturbations in layers 1 and 4 respectively. As expected the response to deeper perturbations occurs later in the trace and occurs over a time span that is associated with the period of the seismic wavelet. The instantaneous response to saturation perturbations is at least one order of magnitude smaller in amplitude and is not shown. Figure 2c shows the time-lapse response to saturation changes in layers 3 and 4 , assuming a uniform porosity of 0.2 . Note that for this case the amplitude response is weaker than in panels a and $b$. When the EnKF method is used to update model variables, use is made of statistical relationships between these model variables and simulated measurements. Figure 3 shows a scatter diagram relating the saturation and seismic amplitude perturbations as shown in Fig. 2c (gray dots). The large scatter resulting from the combination of uncorrelated saturation perturbations in two subsequent layers can be expected to pose problems when covariances need to be estimated between these two quantities, even when the relationship between, e.g., saturation and seismic amplitude is almost linear (the black dots show a linear relationship between saturation and amplitude when saturation in one single layer only is perturbed).
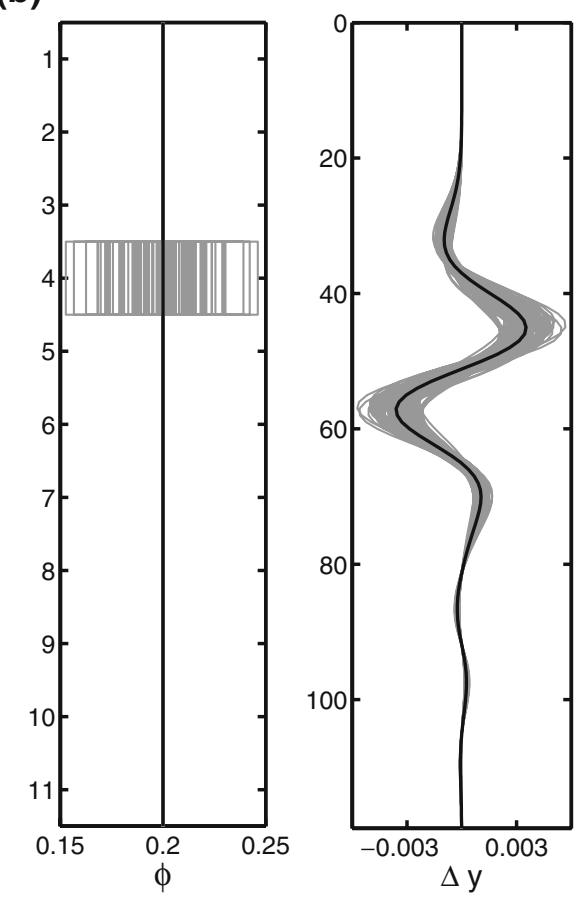

(c)

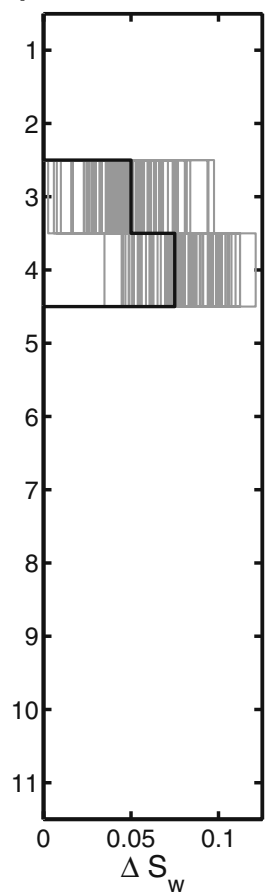

profile and time-lapse seismic response to uncorrelated saturation perturbation in layers 3 and 4. Black line: reference profile. Grey lines: perturbed profiles

\subsection{Ensemble Kalman filter}

The EnKF is a sequential Monte Carlo method that combines fully nonlinear evolution of model and error

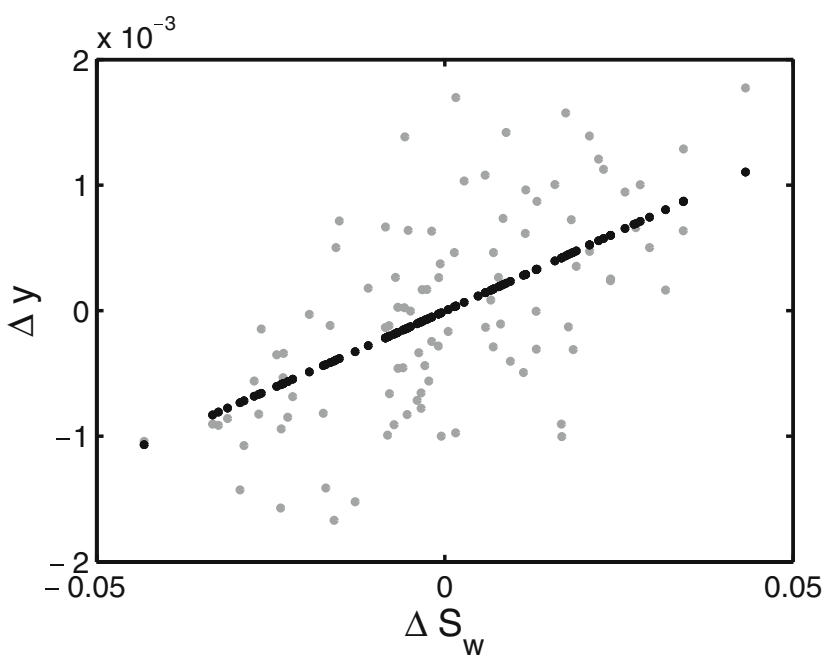

Fig. 3 Saturation change in layer 4 vs seismic amplitude at sample location 45. Black dots: saturation perturbations applied in layer 4 only; grey dots: uncorrelated saturation perturbations applied in layers 3 and 4 
dynamics with a linear updating scheme, based on a low-rank ensemble approximation of the model errorcovariance matrix (e.g., [12]). While sequential filters are traditionally used primarily for state estimation problems, their applicability to parameter and combined state-parameter estimation problems has now been demonstrated in multiple fields. The implementation of the EnKF used here is based on the traditional formulation introduced by Evensen [10] and Burgers et al. [2]. For a measurement equation of the form

$\mathbf{y}=\mathcal{H}\left(\mathbf{x}^{t}\right)+\boldsymbol{\epsilon}$,

where $\mathbf{y}$ is the measurement vector of length $m, \mathbf{x}^{t}$ is the true state vector of length $n, \mathcal{H}$ is the measurement operator, and $\epsilon$ is a random measurement error vector, the EnkF update equation takes the form

$\mathbf{A}_{a}=\mathbf{A}+\mathbf{P}_{x, y}\left[\mathbf{P}_{y, y}+\mathbf{R}\right]^{-1}[\mathbf{Y}-\mathcal{H}(\mathbf{A})]$.

In this equation $\mathbf{A}=\left(\mathbf{x}_{1}, \ldots, \mathbf{x}_{N}\right)$ is a matrix with $N$ ensemble forecast (prior) state vectors as its columns, $\mathbf{P}_{y, y}=\mathcal{H}(\mathbf{A})^{\prime} \mathcal{H}(\mathbf{A})^{\prime T} /(N-1)$ is the ensemblebased error-covariance matrix of simulated measurements, $\mathbf{P}_{x, y}=\mathbf{A}^{\prime} \mathcal{H}(\mathbf{A})^{\prime T} /(N-1)$ is the ensemble-based cross-covariance matrix of simulated states and measurements, $\mathbf{R}$ is the measurement error-covariance matrix, and $\mathbf{Y}=\left(\mathbf{y}+\boldsymbol{\epsilon}_{1}, \ldots, \mathbf{y}+\boldsymbol{\epsilon}_{N}\right)$ is the ensemble matrix of perturbed measurement vectors. Primes indicate anomalies with respect to the ensemble mean, and subscript $a$ and denotes the analysis (posterior).

There are two approximations made in the EnKF which may have consequences in practical cases: (1) the update is based on second order statistics only (i.e., means and covariances), and (2) these covariances are computed from a finite size ensemble. The first approximation implies that third and higher order moments of the joint probability density function (PDF) of the model variables (including state variables and simulated observations) are neglected, which makes it difficult to maintain non-Gaussian a priori statistics of property distributions. There are currently no efficient assimilation methods available for large geophysical problems which address this approximation. Non-linear filters, such as particle filters, as well as Markov Chain Monte Carlo methods, remain too computationally demanding, while methods that attempt to conserve higher-order statistical moments in the joint PDF during the model adjustment (e.g., [31]) are still in an early stage of development. Some iterative approaches have been proposed that attempt to address the issue of non-Gaussian PDFs by looking for a, generally local, maximum likelihood solution.
The second approximation, i.e., the use of a limited number of model realizations, while making the problem computationally tractable, introduces errors in the covariance estimates, most importantly spurious correlations which suggest the presence of non-existing relationships between variables, leading to incorrect updates. Furthermore, it has been shown [39], that such errors tend to lead to systematic underestimation of model error variances, and ultimately to ensemble collapse, which for ensemble filters normally results in filter divergence, that is, a growing tendency for the model trajectory to diverge from the measurements. Experience has shown that similar problems are likely to be encountered when large numbers of relatively accurate data are assimilated, due to loss of rank in the ensemble, i.e., a further reduction in the effective ensemble size. Problems can furthermore be encountered when the initial ensemble spread, or dynamic growth rates are small. Several solutions are available to avoid ensemble collapse. Perhaps the most common solution is localization, which addresses the presence of spurious correlations directly. This will be discussed in more detail in the next section. A second solution is inflation (see, e.g., [1]), which simply means artificially increasing the spread in the ensemble by multiplying the ensemble anomalies, i.e., the difference between each individual ensemble realization with the ensemble mean, by a factor greater than 1 . An alternative solution is the use of so-called square-root schemes which obtain an analysis by factorization of the theoretical analysis covariance matrix. A review of such methods is provided by Tippett et al. [36]. A variation of these solutions is the Deterministic EnKF [28] which is based on an update equation which ensures over-estimation of the analysis variance. A final solution which has been found to produce improved ensemble spread is the use of iterative methods. This approach will also be discussed in more detail in Section 2.4.

\subsection{Localization}

Localization limits the impact of measurements to selected parts of the model, thereby decoupling to some extent different model regions during the update, and in effect increasing the dimension of the solution space to above the number of ensemble members. At the same time, by limiting the impact of measurements to model parts which are presumably most strongly correlated with the measurements, the impact of spurious covariances between measured variables and state variables (resulting by chance due to the finite ensemble size) is reduced. Houtekamer and Mitchell [21] and Hamill et al. [18] both proposed localization of 
the ensemble-based error-covariance matrix of model states $\mathbf{P}_{x, x}$ by taking the elementwise product with a correlation function $\mathcal{C}$ with local support. Common definitions for $\mathcal{C}$ are those suggested by Gaspari and Cohn [16], or by Furrer and Bengtsson [14]. The latter one contains a dependence on ensemble size, leading to reduced localization ranges for smaller ensemble sizes. Assuming that most types of measurements have compact support much smaller than the localization range, Houtekamer and Mitchell [21] then suggested to apply the localization to the ensemble covariance matrix of simulated measurements, and to the matrix of ensemble covariances between state variables and simulated measurements, rather than to the covariance matrix of the simulated states. This removes the need for explicit calculation of the model error-covariance matrix, which is normally very large, and also allows for the use of localization with non-linear measurement operators. The resulting EnKF update equation becomes

$$
\mathbf{A}_{a}=\mathbf{A}+\left(\mathcal{C}_{x, y} \circ \mathbf{P}_{x, y}\right)\left[\mathcal{C}_{y, y} \circ \mathbf{P}_{y, y}+\mathbf{R}\right]^{-1}[\mathbf{Y}-\mathcal{H}(\mathbf{A})]
$$

In history matching applications (e.g. [3], and also this study) the localization is sometimes applied to the Kalman gain instead,

$$
\mathbf{A}_{a}=\mathbf{A}+\mathcal{C}_{x, y} \circ\left(\mathbf{P}_{x, y}\left[\mathbf{P}_{y, y}+\mathbf{R}\right]^{-1}\right)[\mathbf{Y}-\mathcal{H}(\mathbf{A})] .
$$

Chen and Oliver [3] found that for production-type data this practise may result in artifacts when the localization range is comparable to a single well pattern (i.e., typical producer-injector distance). The matrix $\mathcal{C}_{x, y}$ has dimensions $n \times m$, where $n$ is the model state vector length, and $m$ is the number of measurements. Localization can be applied to selected data types only, e.g., seismic data, in which case only the corresponding $m_{s}$ columns are different from 1 .

While the function $\mathcal{C}$ typically depends only on distance, alternative approaches are available for model simulators which support streamlines. Streamlinebased localization for production data was discussed by Devegowda et al. [6]. This approach limits updates to model grid regions which are connected to a particular well by streamlines. The relative merits of distancebased vs streamline-based localization for the incorporation of seismic data were studied by Trani et al. [37]. Synthetic 3D examples for a single five-spot well pattern showed that both types of localization help to better assimilate time-lapse seismic data, and to improve the quality of the permeability estimation, the history match and the production forecast compared to the case where no localization is applied.

\subsection{Iteration}

Several iterative methods have been proposed in the literature with the aim of addressing non-linear effects associated with the estimation of non-Gaussian properties. Early work on the iterated Kalman Filter was reviewed by Jazwinski [22]. Recent ensemble extensions to this idea for reservoir applications were pursued by, e.g., Wen and Chen [41] and Reynolds et al. [27]. The method used here was proposed by Gu and Oliver [17] and coined the Ensemble Randomized Maximum Likelihood Filter (EnRML). It combines the ensemble approach, allowing for the representation of model uncertainty, with a Gauss-Newton type scheme to minimize model-data differences. As it is an iterative filter, only static parameters are updated while consistent dynamic state variables are obtained by simulation of the resulting updated models. Gu and Oliver [17] found that, when observations were used which are strongly non-linearly related to state parameters, dynamic state updates were much improved, while also the ensemble mean and variance were more consistent with the theoretical expected values. The EnRML update step including localization of the Kalman gain can be written as

$$
\begin{aligned}
\mathbf{A}_{l+1}= & \beta \mathbf{A}+(1-\beta) \mathbf{A}_{l} \\
& +\beta \mathcal{C}_{x, y} \circ\left(\mathbf{P}_{x, y}^{*}\left[\mathbf{P}_{y, y}^{*}+\mathbf{R}\right]^{-1}\right) \\
& \times\left[\mathbf{Y}-\mathcal{H}\left(\mathbf{A}_{l}\right)+\mathbf{G}_{l}\left(\mathbf{A}_{l}-\mathbf{A}\right)\right],
\end{aligned}
$$

where $\beta$ is an adjustable step length. $\mathbf{G}_{l}$ is the linear sensitivity of the measurement to the model parameters at iteration $l$ with dimensions $m \times n$, calculated at each iteration from the ensemble as

$\mathbf{G}_{l}=\mathcal{H}\left(\mathbf{A}_{l}\right)^{\prime}\left(\mathbf{A}_{l}^{\prime}\right)^{+}$,

where primes again indicate anomalies with respect to the ensemble mean, and + indicates the generalized inverse, computed here by singular value decomposition. It has been observed that this is the computationally most demanding step in the procedure when seismic data is used, since both the number of observations, as well as the number of state vector elements will be very large. The asterisks in Eq. 7 indicate that the covariances are computed using the linearized measurement operator, e.g.,

$\mathbf{P}_{y, y}^{*}=\left(\mathbf{G}_{l} \mathbf{A}^{\prime}\right)\left(\mathbf{G}_{l} \mathbf{A}^{\prime}\right)^{T} /(N-1)$.

Note that for parameter or initial value estimation problems computation of $\mathcal{H}\left(\mathbf{A}_{l}\right)$ involves running the 
simulator from the initial time to the update time, as well as evaluating the measurement operator on the dynamic state. We currently use three stopping criteria, two of which are based on an evaluation of the data mismatch,

$\left.J_{l}^{d}=\sum_{j=1}^{N}\left(\mathbf{Y}_{j}-\mathbf{D}_{j, l}\right)^{T} \mathbf{R}^{-1}\left(\mathbf{Y}_{j}-\mathbf{D}_{j, l}\right)\right)$

where $J_{l}^{d}$ is the data mismatch part of the objective function at iteration $l$, and $\mathbf{Y}_{j}=\mathbf{y}+\boldsymbol{\epsilon}_{j}$ is the perturbed observation vector for ensemble member $j$. The iteration process is stopped if at least one of three conditions is met: (1) $S_{l-1}-S_{l}<\alpha S_{l-1}$, where $\alpha$ is some small number, (2) $S_{l}<m$, or (3) $l>l_{\max }$.

\subsection{Time-difference data}

Special attention has to be paid to correct treatment of time-difference data such as time-lapse seismic data. Using a Bayesian formulation, it was demonstrated by Skjervheim et al. [32] that, since the simulated timelapse data at time $t_{j}$ depend both on the state at time $t_{j}$ , as well as on the state at the initial time $t_{0}$, differences should be calculated with respect to the smoothed estimate for the state at the initial time $t_{0}$. The smoother solution can be obtained by adding the initial state to the state vector $\mathbf{x}$, and updating it at every time $t_{i}$, $0<i<j$ at which measurements are assimilated. There are two possible options here: we can compute the smoother solution for the initial ensemble state and simulate the corresponding seismic measurements, or we can simply compute the smoother solution for the simulated seismic measurements at the initial time. In order to save computational effort, we apply the second option here, but we do not claim that this option is necessarily the best. In particular when iteration with the EnRML is used it is not obvious what the optimal method is. Note that when the EnRML is used, the dynamic states are generally not updated, so that if the initial saturation and pressure are assumed to be known, the only uncertain parameter pertaining to the seismic response at the initial time is porosity. This will typically already be included in the list of parameters to be updated. However, if the initial saturation distribution is unknown, it should also be updated. Note furthermore that, even though permeability will not affect the seismic response directly, it will generally be related to porosity by a porosity-permeability relation and should therefore also be updated for consistency. Skjervheim et al. [32] recommended that the ensemble should additionally be conditioned to the initial 3D seismic data. We will present an experiment that investigates this issue.

\section{Experiments}

\subsection{D five-spot model}

We start our experiments with a simple case that illustrates the potential for model improvement by use of non-production type data. We consider a square, $2 \mathrm{~m}$ thick and $700 \mathrm{~m}$ in width, flat oil reservoir with a water injection well in the middle and four producing wells in the corners. The reservoir is modeled by a single layer of $21 \times 21$ grid cells. The only uncertain property of the reservoir is its grid cell permeability (porosity is 0.2 everywhere). In order to account for the uncertainty in the knowledge of the reservoir, an ensemble of 200 possible realizations is generated based on the true geostatistics which are assumed known. The true permeability values lie between 25 and $600 \mathrm{mD}$, with a mean $\log _{10}$ value of 2.23. As an example, Fig. 4 shows four randomly picked permeability realizations from the ensemble, and Fig. 6a shows the true permeability. Bottom-hole-pressures (BHP), and water and oil rates are available for all wells every 50 model days. Grid
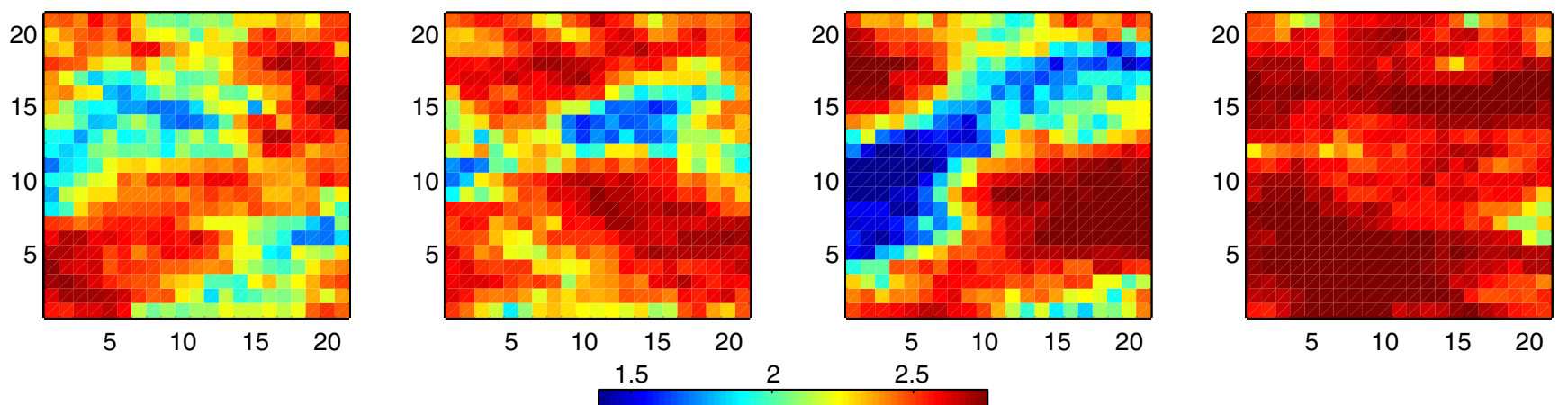

Fig. 4 Log permeability of four randomly picked realizations for the 2D reservoir 
saturation values, used here as a proxy for seismic data, are available at days 150 and 450 for each grid cell. Whenever measurements are available they are used to update the model ensemble using the Ensemble Kalman Filter. No localization is used. The updated ensemble is then simulated forward in time up to the next measurement time and the process is repeated. After the final update at day 500 all models are run forward up to day 1,500, providing a prediction of future production given prescribed well constraints (an injection rate of 1,090 barrels per day (BPD) and a BHP of $250 \mathrm{Bar}$ for the producers). Since this is a synthetic case, measurements can be generated by simulation of the known true model, and the performance of the models can be verified by comparison with the true production profiles. In order to add realism to this case, the assimilated measurements are perturbed with random noise: 2.5 Bar for BHP, $10 \%$ relative error for rates with a minimum of $2 \mathrm{BPD}$, and 0.05 saturation units for the seismic data.

Figure 5 shows time series of the water cut (WCT) of the four producers for three different cases. The first case (a) is an ensemble run without any conditioning to data. The ensemble spread results from the uncertainty in the permeability, leading to strongly varying production profiles, with the water breakthrough time varying up to a few hundred days between realizations. The second case (b) is an EnKF run with conditioning to well data only. The history match has reduced the uncertainty, leading to more reliable predictions, although significant ensemble spread remains for wells P1 and P4. The third case (c) is an EnKF run with conditioning to both production data and proxy seismic data. As expected, the incorporation of saturation information has further improved both the history match and the prediction. Note especially that the history match has improved the timing of the water-breakthrough, even when it occurs long after the final update. Figure $6 \mathrm{~b}$ to d show the ensemble-mean permeability fields corresponding to the three cases. The final ensemblemean estimate of permeability after the history match is far closer to the true field than the initial estimate, with more detail being recovered whenever more information from measurements is incorporated. It can be concluded from these simple experiments that, when used in an appropriate manner, information from seismic data can be expected to contribute in a quantitative manner to the improvement of reservoir models. In the following section we will discuss this quantitative approach in more detail, and add complexity by

\section{P1}

(a)

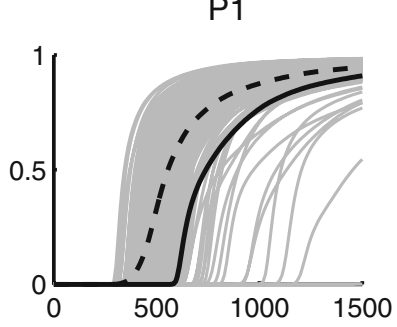

(b)

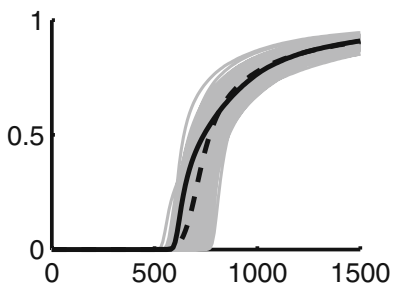

(c)

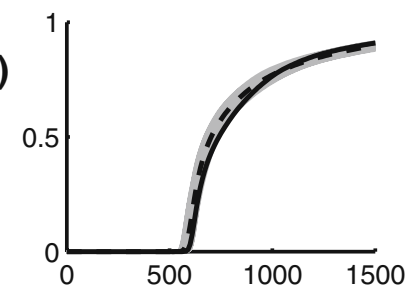

P2
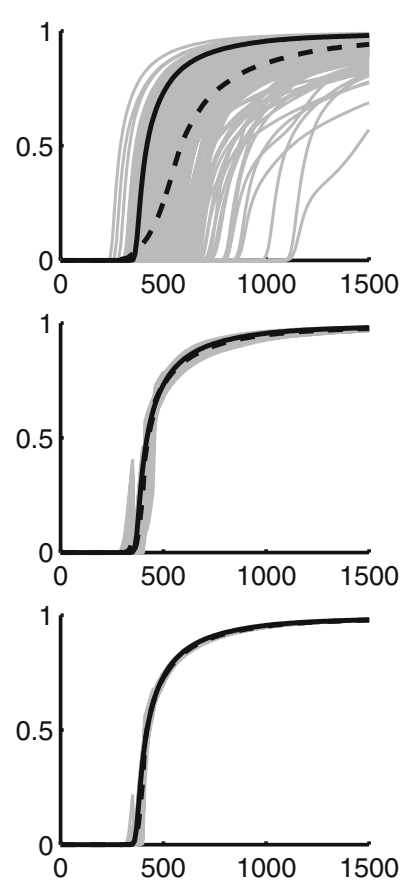
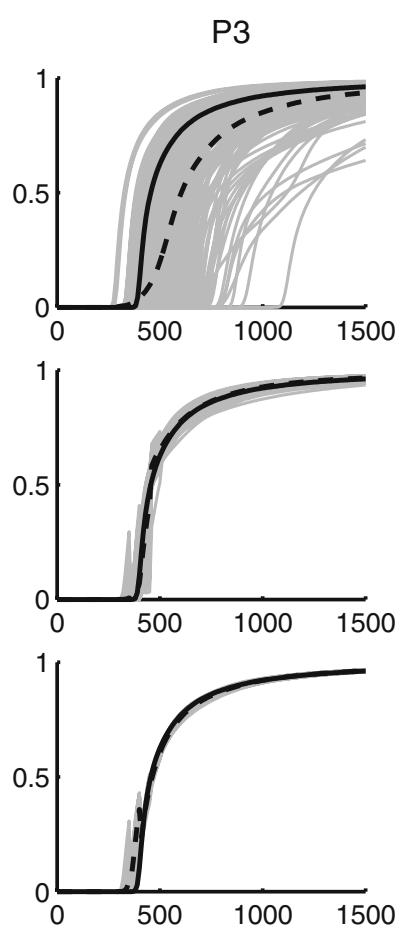

P4
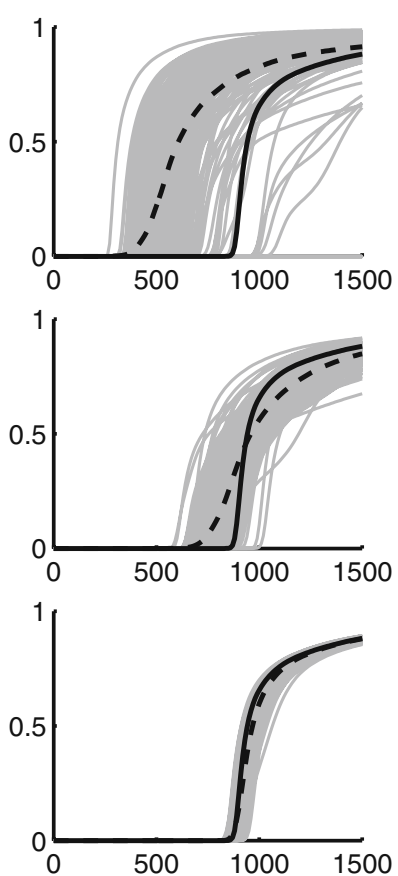

Fig. 5 Profiles of the water cut (WCT) of producing wells P1 to P4. a unconditioned ensemble run, b EnKF run based on production data only, $\mathbf{c}$ EnKF run based on production data

and proxy seismic. Grey solid lines: profiles for individual model realizations; dashed black line: ensemble mean; solid black line: true profile 
(a)

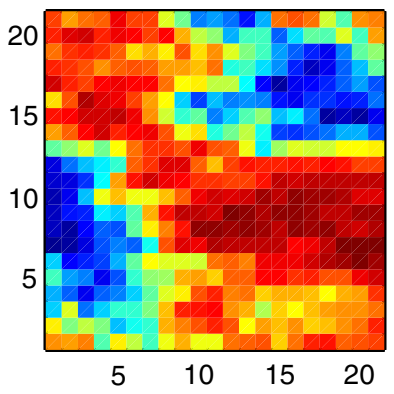

(b)

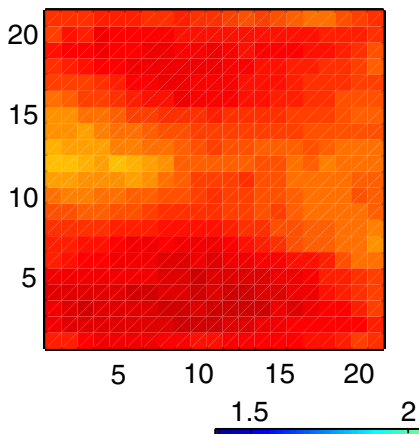

(c)

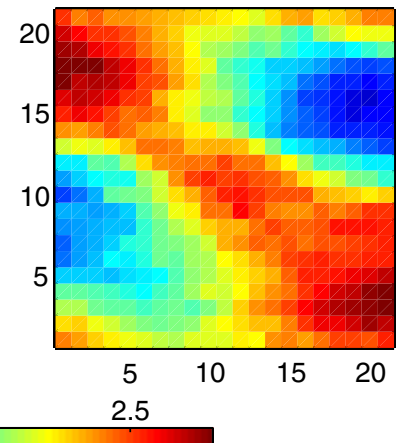

(d)

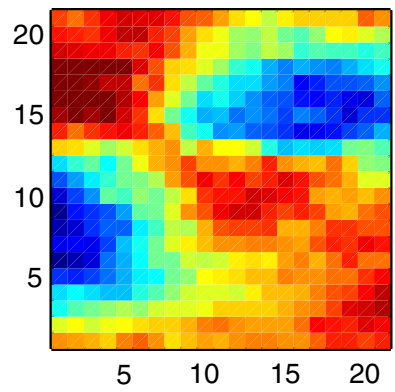

Fig. $6 \log _{10}$ permeability for the 2D reservoir; from left to right: a the truth, $\mathbf{b}$ the initial ensemble mean, c ensemble mean conditioned to production data, and d ensemble mean conditioned to production and seismic data

considering the general 3D case in combination with the seismic amplitude data discussed in Section 2.1.

\subsection{D five-spot model}

In order to investigate the use of the seismic data discussed in Section 2.1, a 3D model will be used in this section which resembles the 2D case discussed above in terms of well configuration and constraints. The model consists of 11, $2 \mathrm{~m}$ thick, layers of $N_{x}=$ $15 \times N_{y}=25$ square grid cells of $33 \mathrm{~m}$ width each. An ensemble of 50 model realizations and a truth realization were generated by random drawing from the same permeability-porosity distribution. Example permeability realizations are depicted in Fig. 7a and $\mathrm{b}$, and a corresponding porosity realization in Fig. 7c. The top two layers have identical properties. There are two zones, consisting of layers 3 and 4, and of layers 7 to 11 respectively, with relatively high porosity and permeability. The well configuration for this model is again a five-spot, with a slightly deviated central injector well and vertical producers positioned in the corners. All wells are perforated in layers 2 to 8 , and the injector is perforated in layers 2 to 9 . All realizations are simulated for 1500 days under a prescribed constant rate constraint of $5435 \mathrm{BPD}$ for the injector and a constant BHP constraint of 250 Bar for the producers. The model is identical to that used by Trani et al. [37] who investigated the impact of localization for a case in which saturation and pressure changes, as obtained from an inversion of time-lapse seismic AVO data, were assimilated with the EnKF. Pressure and rate data are available every 50 days up to day 500 and seismic data every 150 days up to day 450 , including a base survey before the start of production. Seismic traces are obtained at regular intervals of 3 and 5 grid cells in $x$ and $y$ direction respectively, and the traces are subsequently sub-sampled in time, retaining every 5th sample, resulting in a total of $25 \times 17=425$ seismic measurements from each survey. Examples of the simulated seismograms corresponding to a single grid column of the model are depicted in Fig. 8. Figure 8a shows the porosity for the grid column as well as the seismic amplitude and saturation for that column after simulating the ensemble for 700 days. Superimposed on the ensemble results are the values from the true reservoir. Several observations can be made. As also shown in Fig. 7, permeability and porosity vary rather strongly between layers as well as across the ensemble. This is reflected in the simulated saturation as well as the seismic response for this grid column, which shows large spread in the amplitude data. The time-lapse seismic response to the reservoir properties will be rather complicated due to the influence the property field has on the dynamic variables saturation and pressure. Since for multi-phase flow the dynamic response is not linear in each of these properties, the influence of the porosity profile, for example, may possibly not be eliminated from the time-lapse response. In the following section we will further investigate the relationship between the simulated seismic amplitudes and reservoir properties and dynamic variables. As a prelude to the history matching experiments, Fig. 8b shows an example of a porosity update for a single grid column. For this example, the porosity was assumed to be the only uncertain variable, with no prior knowledge on the vertical distribution. The prior ensemble layer porosities are drawn from a Gaussian distribution with a mean value of 0.15 and standard deviation of 0.6. Measurements of seismic amplitude, containing noise with a standard deviation of 0.02 were sub-samples from the seismic trace (black dots in the left panel). A single update with the EnKF 


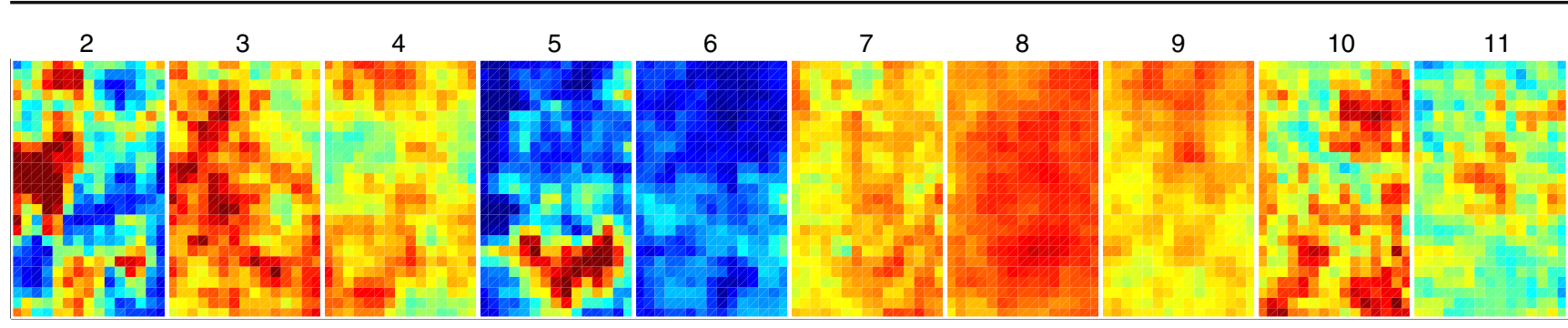

(a)
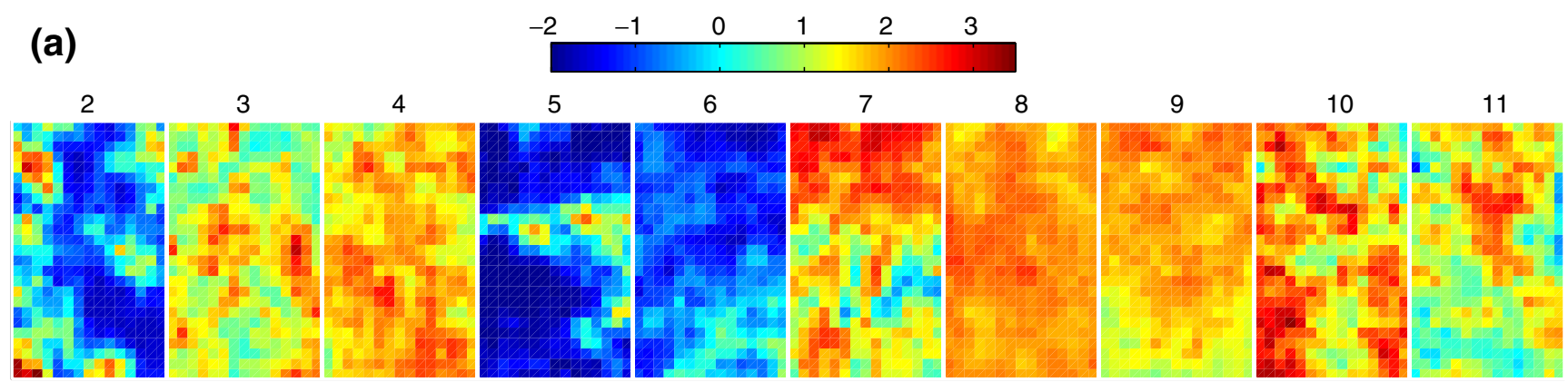

(b)
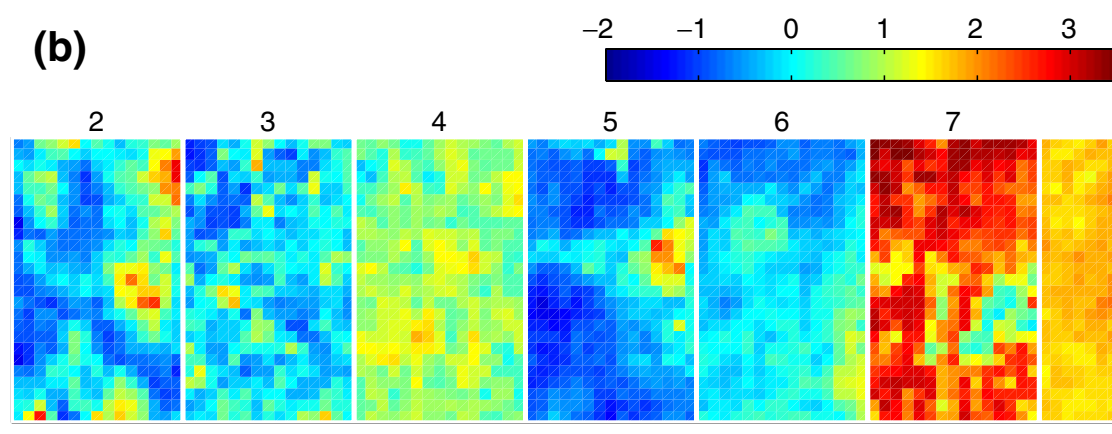

8
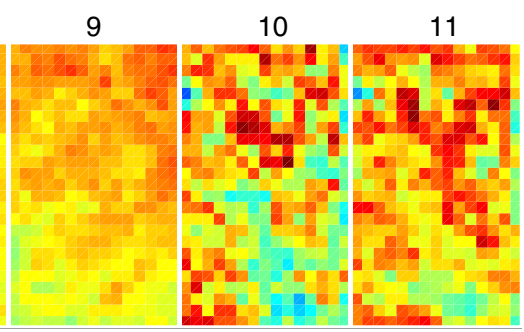

(c)

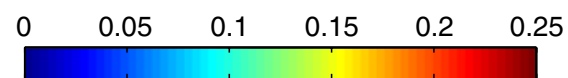

Fig. 7 3D five-spot model used for history matching with synthetic seismic trace data. a, b Two random $\log _{10}$ permeability realizations in layers 2 to 11 . $\mathbf{c}$ Porosity corresponding to the realization shown in $\mathbf{b}$

results in the dark grey lines and dots. The general character of the column with high and low porosity zones can be seen to be reconstructed to some extent, supporting our expectation that the seismic amplitudes may be able to improve the vertical resolution of the reservoir property distribution. This will also be further investigated in the next section.

\subsubsection{Ensemble-based cross-covariances}

As discussed in Section 2.2, ensemble methods for model updating rely on empirical statistical relationships in the form of covariances between simulated measurements and state vector elements (either static parameters or dynamic variables). In this section we will investigate the character of these covariances for the seismic data discussed in Section 2.1.
In order to examine the extent to which the amplitude samples in the seismic trace are able to discriminate reservoir characteristics in the vertical, as well as laterally, Fig. 9 shows the normalized cross-covariances between seismic amplitude and state vector components (porosity and saturation are shown) after running the ensemble realizations with the reservoir simulator for 700 days. Each figure shows a composite of $9 \times 9$ sub-panels corresponding to layers (layer numbers are indicated at the top). The colors represent the magnitude of the covariance between model variables defined in grid cells for that layer and the seismic amplitude for a certain sample in the trace. The sample number is indicated to the left of the panel (the full trace is 120 samples long). The $(x, y)$ location of the seismic trace is indicated by the dot. The covariances were normalized by the ensemble variance of the seismic 
(a)

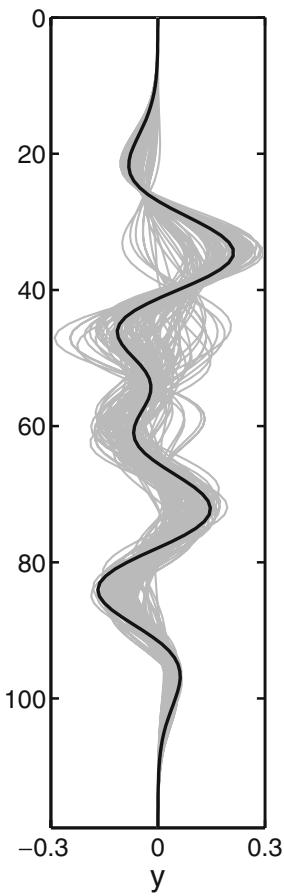

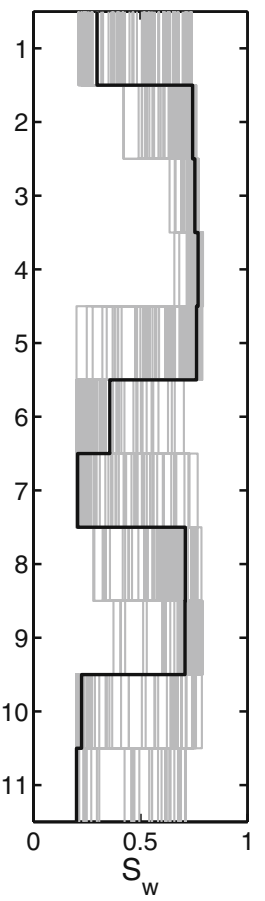

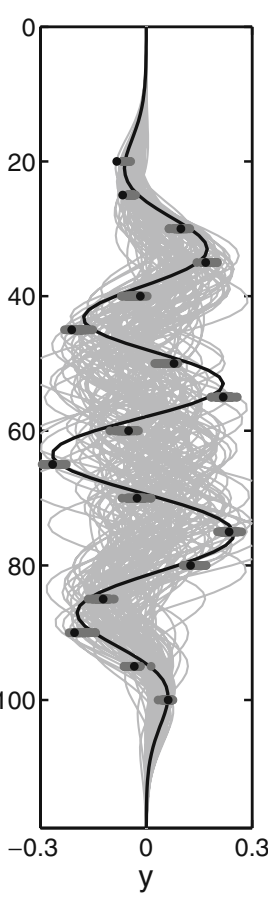

(b)

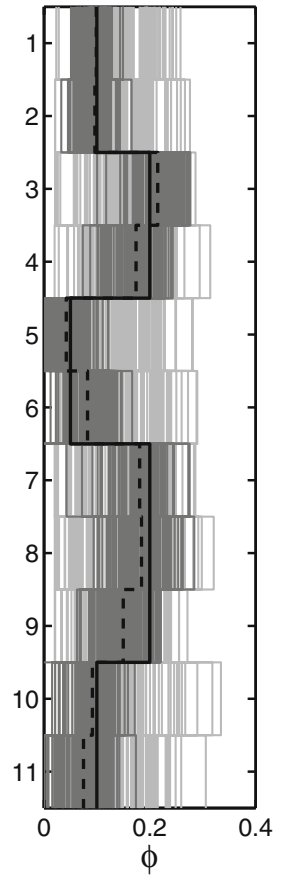

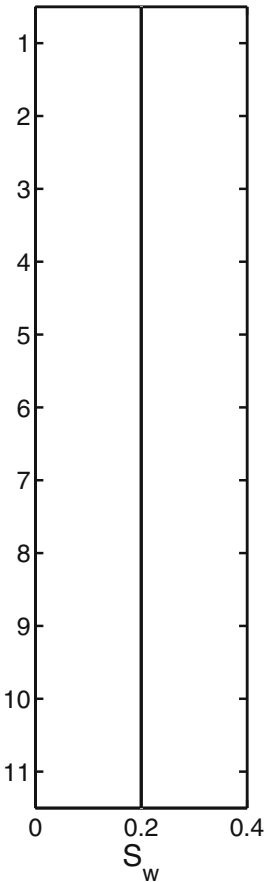

Fig. 8 a Seismic amplitude, porosity and water saturation for a single grid column $(x=8, y=12)$ of the $3 \mathrm{D}$ reservoir model after 700 days of production for all model realizations. Solid black line: truth model. Grey lines: ensemble realizations. b Synthetic example of porosity update based on seismic data. Black solid

amplitude and can therefore also be interpreted as the Kalman gain given zero measurement error, or as the magnitude of the corresponding variable update for a unit innovation. The top two panels, Fig. 9a and b, show the ensemble cross-covariances between porosity and seismic amplitude for two different seismic traces, the first being located close to the injector well, and the second relatively close to the bottom-left producer well.

The spatial extent of the observed patterns may seem unrealistic from the point of view of the information contained in a single seismic trace. However, the covariance patterns are influenced by the spatial scales imposed on the porosity realizations as well. Since the EnKF is based on statistical relationships resulting from an ensemble of realizations, model-wide updates could in principle result from a single observation, such as a single sample from a seismic trace. The spatial extent of the updates may be limited by the use of localization. It is not immediately obvious what the appropriate localization range should be, however, as fairly strong covariances are observed to extend throughout at least a single five-spot well pattern.

Several additional observations can be made. Covariances tend to be strong for the top layer and tend line and dots: truth and measurements, light grey lines: ensemble realizations and simulated data, dark grey lines and dots: updated realizations and simulated data, dashed line: updated ensemble mean. See text for further explanation

to become weaker for deeper layers. This is related to the impedance contrast which is strongest between the overburden and top reservoir, and relatively weaker between individual reservoir layers. A similar effect can be observed for the bottom layer, for which the impedance contrast with the underburden is again relatively strong, and for contrasts between high- and lowporosity zones. A second effect that can be observed is that spatial features in the deeper layers become progressively clearer in covariances with samples which are located later in the trace. This is due to the delay in travel time, i.e., the seismic signal arrives later at interfaces between the deeper layers (compare Fig. 2a and $b$ ). A factor contributing to the magnitude of the covariances is the ensemble spread in the reservoir properties for individual layers. The fact that this is relatively large for the bottom layers as well as for layer 7 (see Fig. 8) also contributes to the larger values for these layers.

The bottom two panels consider cross-covariances between porosity or saturation and time-lapse seismic amplitudes. A still significant covariance is observed with porosity, even though porosity itself does not change over time. It is our interpretation that these 
Fig. 9 Ensemble

cross-covariance between static and dynamic variables and seismic amplitude response after 700 days of production. The amplitude samples vary over the rows from 20 to 100 , while the layers vary with the columns from 2 to $10 . \mathbf{a} \operatorname{Cov}(\phi, y)$ in porosity units for a seismic trace located at

$(x=7, y=13), \mathbf{b} \operatorname{Cov}(\phi, y)$ in porosity units for a seismic trace located at

$(x=5, y=5), \mathbf{c} \operatorname{Cov}(\phi, \Delta y)$ in porosity units for a seismic trace located at $(x=7, y=13), \mathbf{d} \operatorname{Cov}(S, \Delta y)$ in saturation units for a seismic trace located at $(x=7, y=13)$
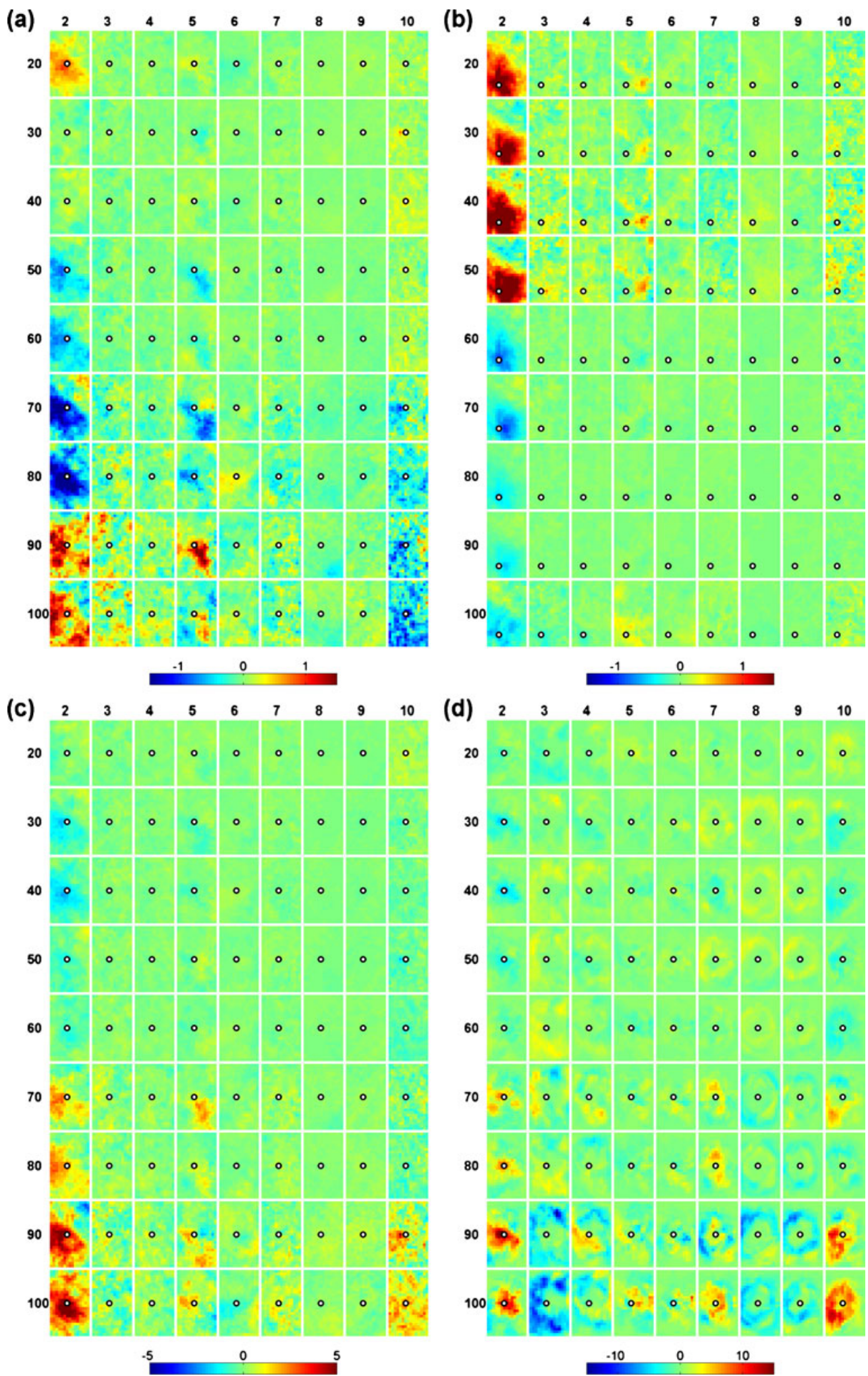

covariances result indirectly through the change in saturation, for which the covariance plot is shown in panel (d). The relevance of this is that one may expect that time-lapse seismic can most likely be used to update time-constant properties. The covariance patterns for saturation are clearly associated with the spreading 
of the water front away from the injector. The sign and position of the resulting saturation update would be different for the different layers, again indicating the potential to improve vertical distributions of model properties and dynamic variables. This assumes of course that the seismic frequency is high enough relative to the thickness of the reservoir.

Based on these figures, we expect that significant information on the vertical distribution of both static properties, such as porosity, and dynamic properties, such as saturation, can be extracted from the seismic by use of an ensemble of model realizations.

\subsubsection{History matching experiments}

In this section several history matching experiments with the $3 \mathrm{D}$ reservoir model will be described. For comparison between the different experiments we will consider the water-cut (WCT) profile for the four producers resulting from running the reservoir simulator for 1,500 days with the updated property fields. As a reference, the results from a simulation of the unconditioned ensemble is shown in Fig. 10a. In each of the panels the root-means-square error (RMSE) in the WCT is given, computed over all ensemble members from the difference with the measurements. We note that this is by far not the only possible measure to judge the success of the history match. Alternatives are, for example, to compute the RMSE between simulated and measured data over the history match period, or to consider only the ensemble mean. Similarly, rather than plotting the ensemble-mean WCT, as we do here, one could instead consider the model realization with the smallest RMSE.

Following the suggestion by Skjervheim et al. [32] we first investigated the impact of incorporating the initial 3D seismic base survey data. Eight iterations with the EnRML were performed to obtain a match between simulated and measured seismic amplitudes consistent with the specified error statistics. A measurement error standard deviation of 0.02 was used for the 3D seismic amplitudes. This is a small fraction of the initial ensemble spread in the predicted seismic amplitudes (see Fig. 8) and is therefore representative of the case in which seismic data has the potential to reduce model uncertainty significantly. When the reservoir and seismic models were subsequently simulated, the seismic response shown in Fig. 11a was obtained after 700 days. This figure can be compared to Fig. 8a. The ensemble mean seismic response has clearly moved closer to the true response. The ensemble spread has also been reduced considerably. Some inconsistency
Fig. 10 a Profiles of water-cut obtained by running the initial (prior) ensemble forward over the combined history match (0-500 days) and forecast (500-1,500 days) periods. In this and the following figures, the true values are indicated by the solid black line, the ensemble mean by the dashed black line, and individual ensemble member profiles by the solid grey lines. The RMSE value is based on the differences between all individual ensemble members and the truth over the complete period, and therefore incorporates both the effects of bias in the mean and ensemble spread. b Same as a but for an ensemble whose mean was additionally conditioned to the initial 3D seismic (a)
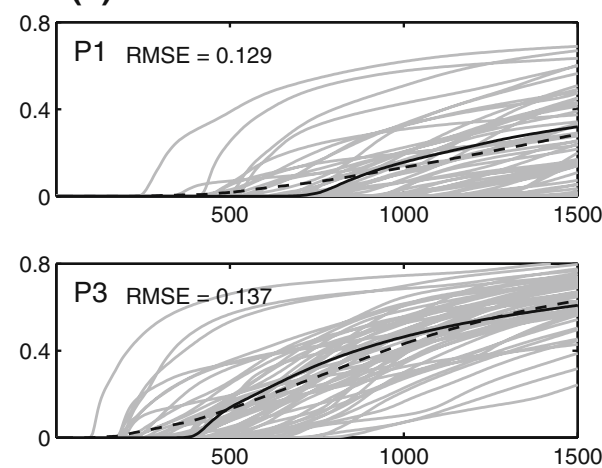

(b)
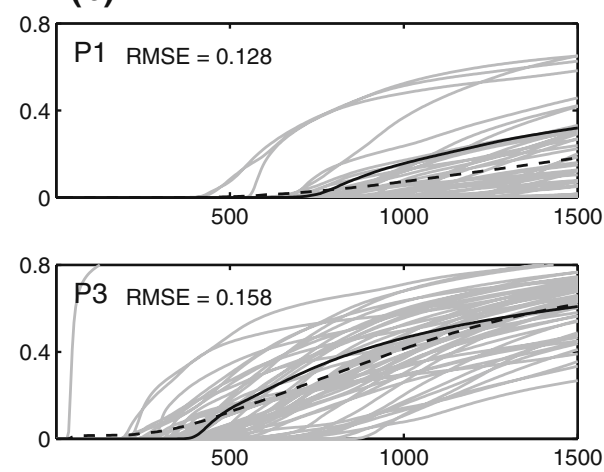
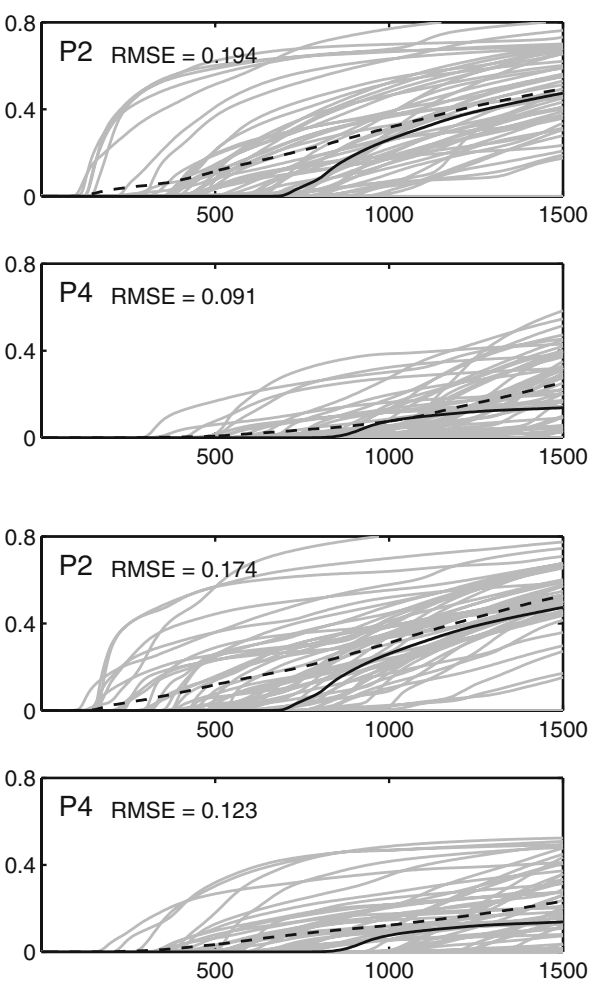


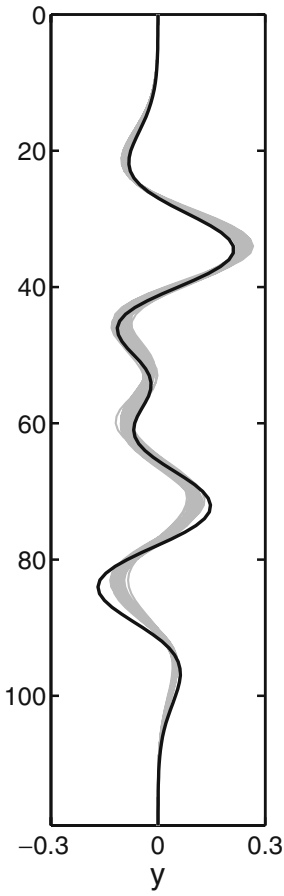

(a)

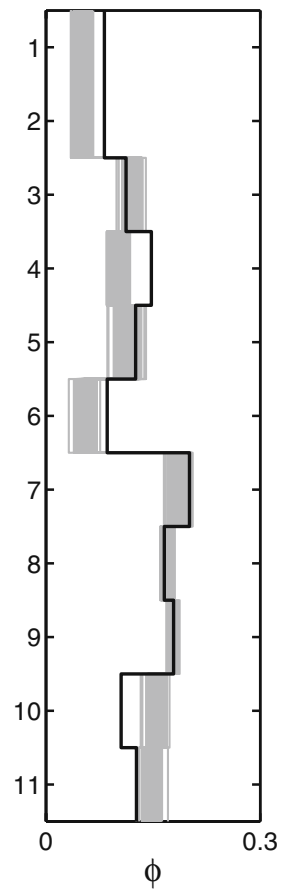

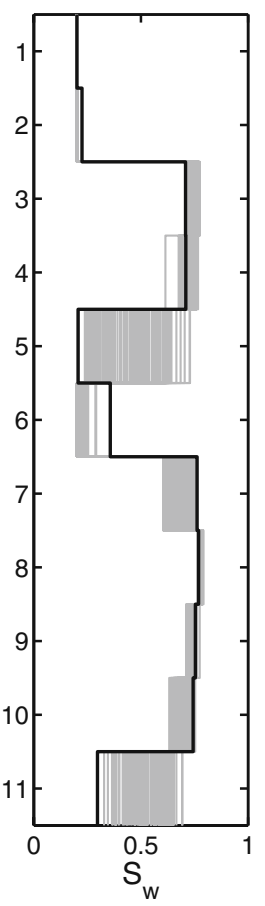

$\mathrm{S}_{\mathrm{w}}$

Fig. 11 Impact of conditioning to initial 3D seismic and inflation. a Seismic amplitude, porosity, and water saturation for a single grid column $(x=8, y=12)$ of the 3D reservoir model after 700 days of production for all model realizations after condi-

between the ensemble and the truth has appeared however, which is most clearly visible in the porosity profile. In order to resolve this mismatch, the iteration process was repeated but in this case only the ensemble mean was updated, after which the initial ensemble spread was restored around the updated mean. The results are shown in Fig. 11b, which again shows the seismic response after 700 days of production, and in Fig. 10b, which shows the water-cut history for a 1,500 day simulation. This ensemble will be further used in some of the history matching experiments described below.

Figure 12 shows the results from two experiments in which only well data (BHP, oil and water rates) were used to history match the ensemble. In both cases the sequential EnKF was used every 50 days to assimilate production data after which the updated ensemble was further simulated to the next update time. No localization was applied. This choice is consistent with the results of Chen and Oliver [3] who found that localization patterns for rate and pressure data should extend beyond a single well pattern such as the five-spot used here. Note that only one well shows water breakthrough during the history match period, and that therefore one can expect to obtain only

(b)
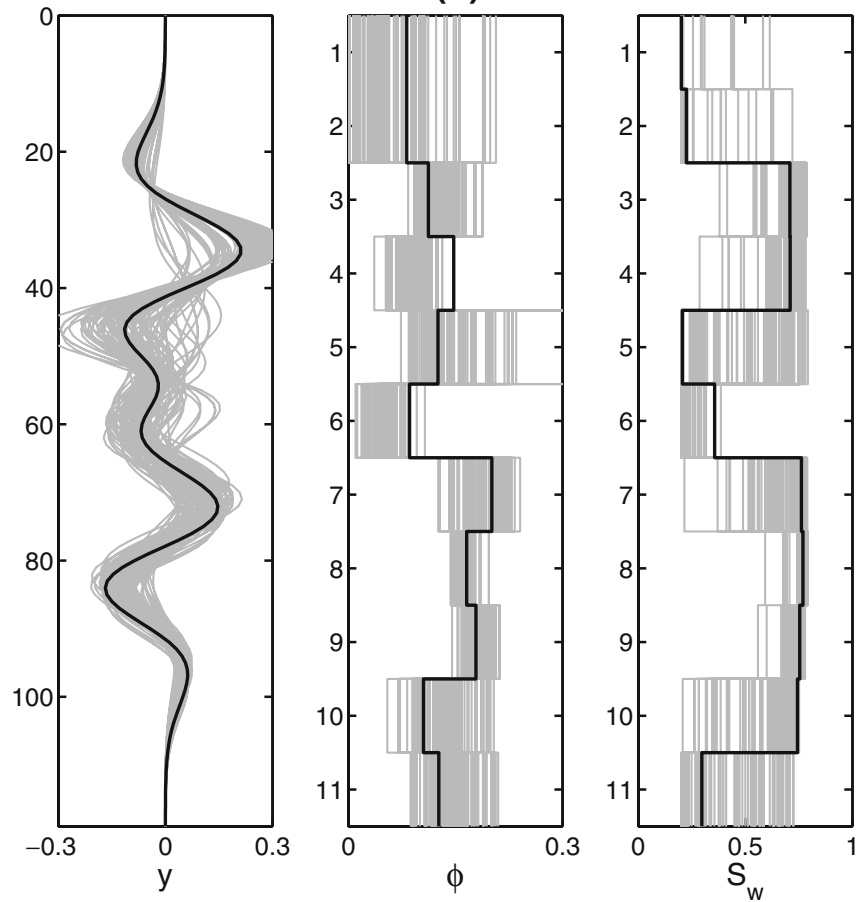

tioning of the model porosity to the initial 3D seismic data with the EnRML. b Same as a but with updating of the ensemble mean only. Solid black line: truth model. Grey lines: ensemble realizations

limited model improvement from the well data alone. Figure 12a shows the WCT profile obtained during the first EnKF run itself, while Fig. 12b shows the WCT profile when the final updated ensemble is rerun from the initial time. This experiment was started from the unconditioned ensemble. In Fig. 12c, on the other hand, the WCT profile from a history match using the same data is shown when the ensemble conditioned to the initial 3D seismic is used. In both cases the resulting profiles are closer to the truth than for the initial (prior) ensemble, but significant deviations from the true profile, as well as large uncertainties, as indicated by the large ensemble spread, remain.

Figure 13a to $d$ show the results from a series of experiments in which, additionally to well data, seismic time-lapse amplitude data were assimilated at days 150,300 , and 450. An error standard deviation of 0.01 was assumed for the seismic amplitude measurements. Figure 13a results from an EnKF run using the same settings as were used to obtain the results in Fig. 12c. As can be observed right away, large deviations have appeared between the ensemble and true WCT profiles, accompanied by a strongly reduced ensemble spread. This is the typical signature of filter divergence. For the 
Fig. 12 Profiles of water-cut resulting from history matching with the EnKF to well data (BHP, oil rate, and water rate). a Profiles obtained during the EnKF run. b Profiles resulting from simulating the updated models over the history match period. c Same as b but for an ensemble whose mean was additionally conditioned to the initial 3D seismic

\section{(a)}
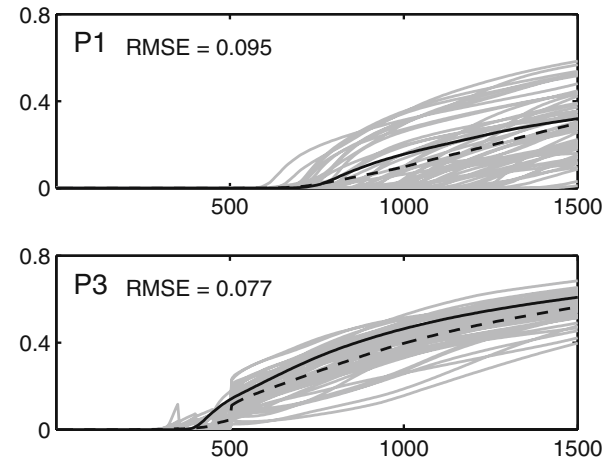

(b)
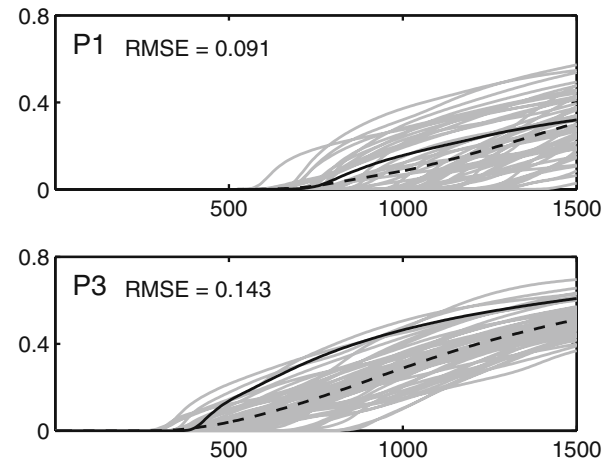

(c)
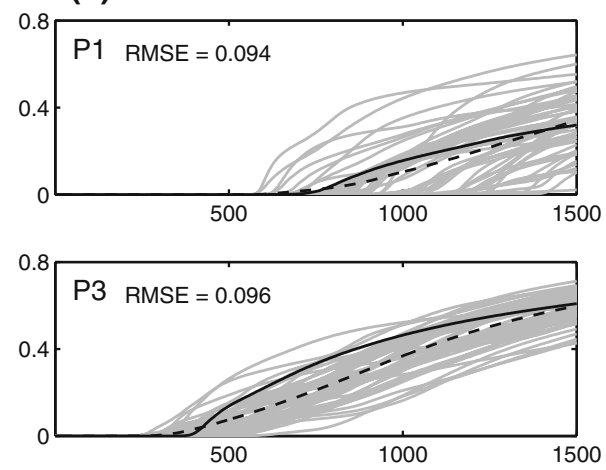
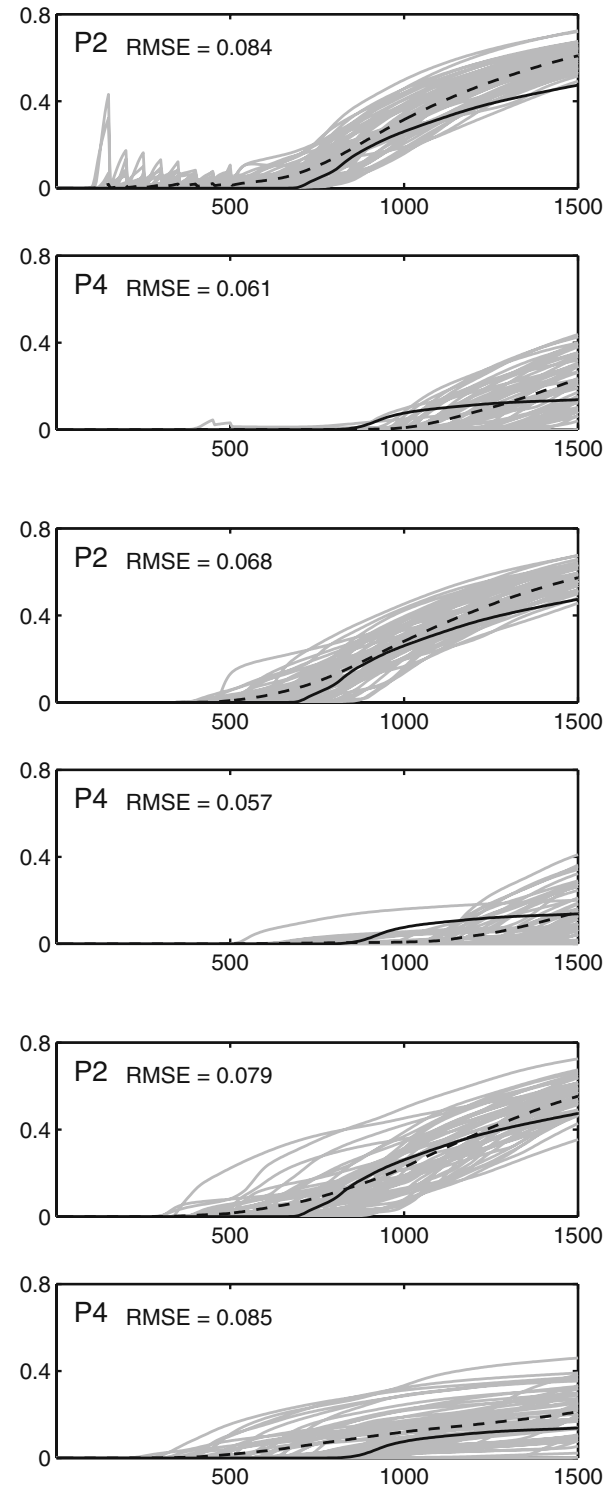

next run, the results of which are shown in Fig. 12b, lateral distance-based localization was applied to each seismic data trace using the localization function suggested by Furrer and Bengtsson [14]. The localization distance was somewhat arbitrarily chosen such that the function $\mathcal{C}$ has a value of 0.5 at the locations of neighboring traces. While the use of localization has significantly helped to restore ensemble spread, large deviations and inconsistencies between the ensemble mean profile and the truth remain. In an attempt to reduce the negative impact of large model-data mismatches, we increased the measurement error variances, contained in the matrix $\mathbf{R}$ for mean innovations exceeding three times the measurement error standard deviation. This leads to the profiles shown in Fig. 12c. The bias between modeled and measured WCT is seen to be somewhat reduced, and ensemble spread has been further increased for at least one of the wells. For the final figure, Fig. 12d, we applied a simple adaptive ensemble inflation procedure, similar to the one used to incorporate the 3D seismic from the base survey. Only when ensemble spread, as defined by the maximum eigenvalue of the model property covariance matrix, reduced by more than $40 \%$ during an update step (which occurred only when seismic data were included), ensemble spread was restored to its value before the update by multiplying the ensemble property anomalies. We did not extensively test for the minimum required value. Although not required for the stability of the simulations, the simulations were 
Fig. 13 a Profiles of water-cut resulting from history matching to production data (oil rate, water rate, and flowing bottom-hole-pressure) and time-lapse seismic amplitude data. Time-lapse differences were computed with respect to the base survey. b Same as a but with localization as described in the text. c Same as b but with selective increase of the measurement error variances in $\mathbf{R}$. d Same as $\mathbf{c}$ but with ensemble inflation as described in the text, taking time-differences with respect to the most recent repeat survey, and restarting from initial time after each seismic update. In all cases, the initial ensemble mean was conditioned to the initial 3D seismic

\section{(a)}
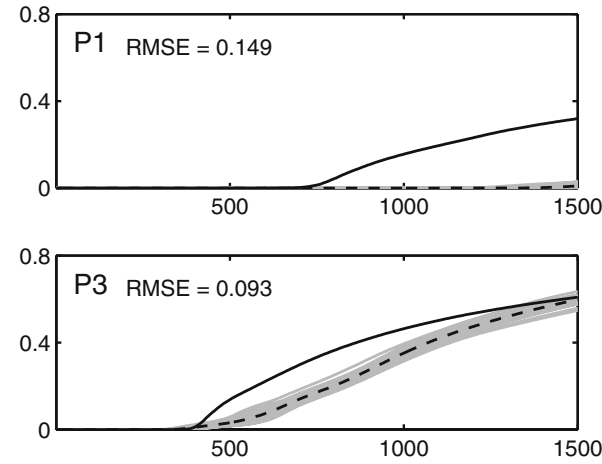

(b)
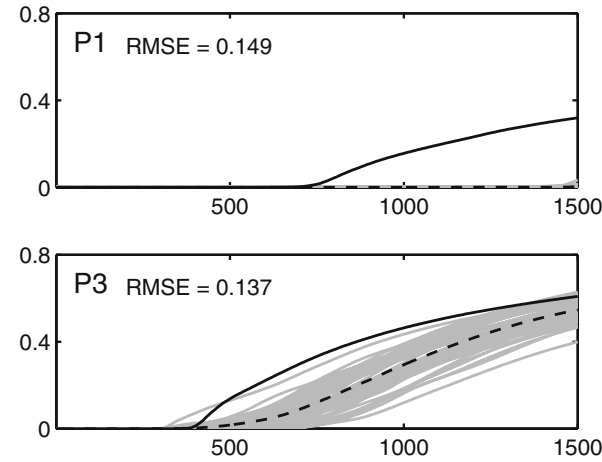

(c)
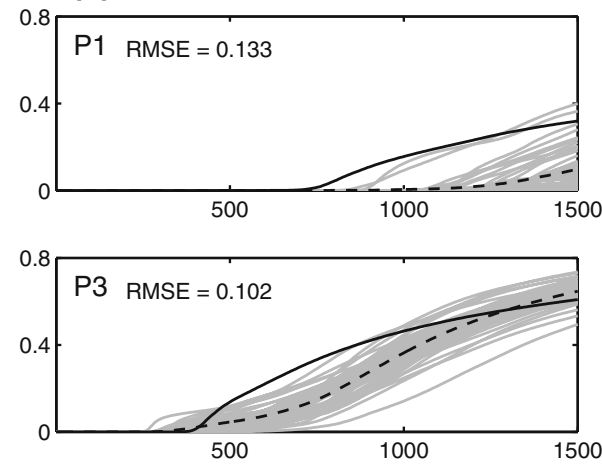

(d)
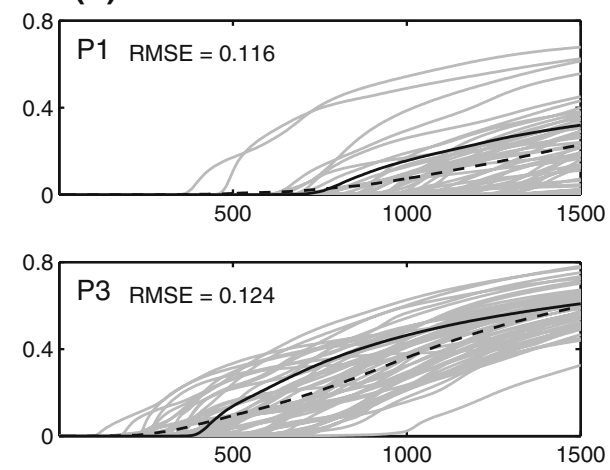
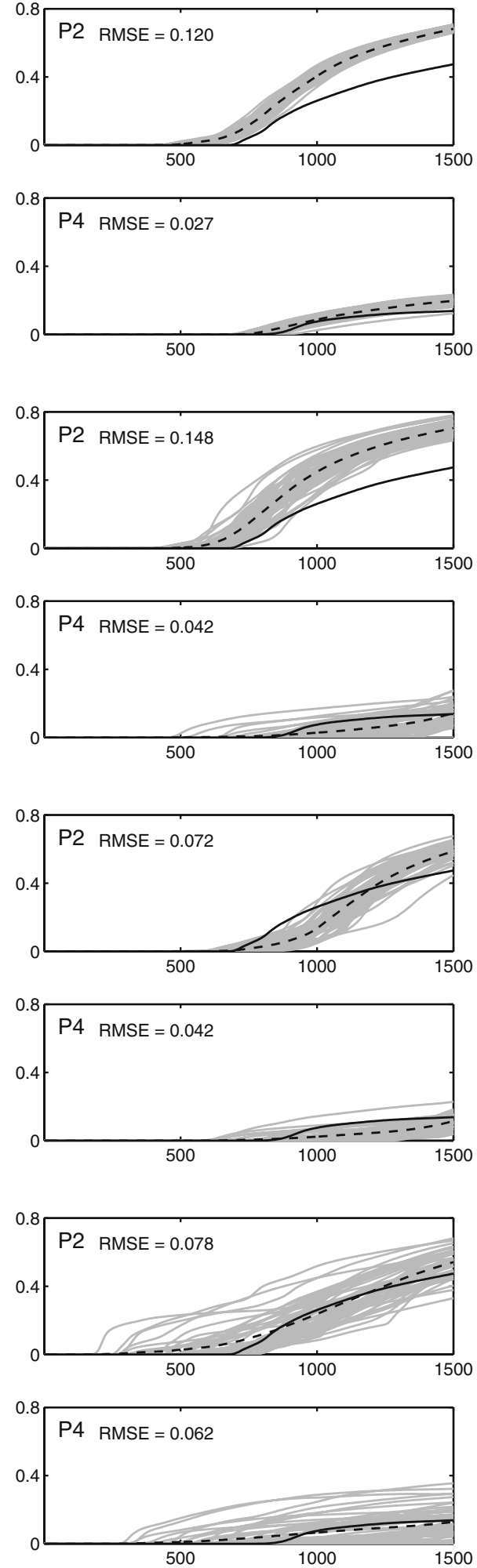

restarted from the initial time after each seismic update, which ensures internally consistent balances between the parameter field and dynamic variables, but comes at the cost of doubling the run time for the seismic update steps. Finally, for this run, time-lapse differences were computed with respect to the most recent survey, 


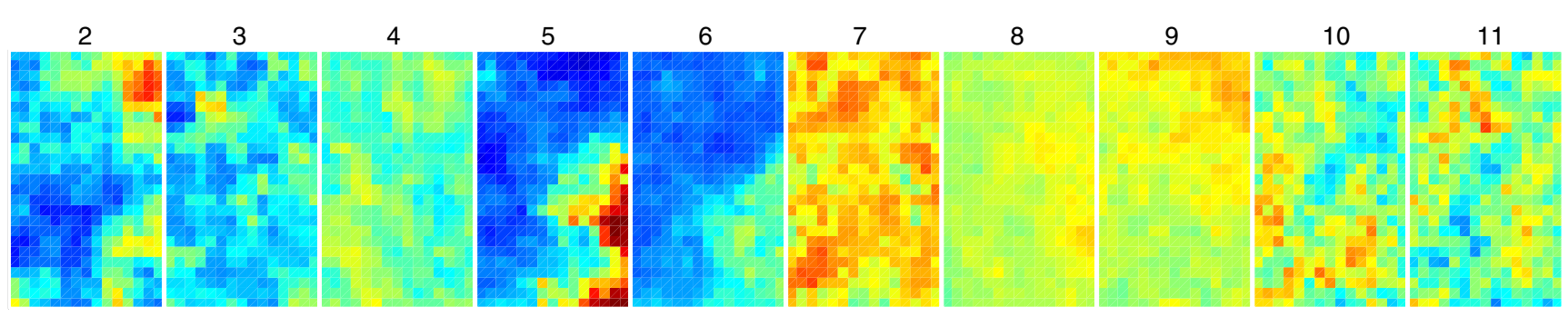

(a)
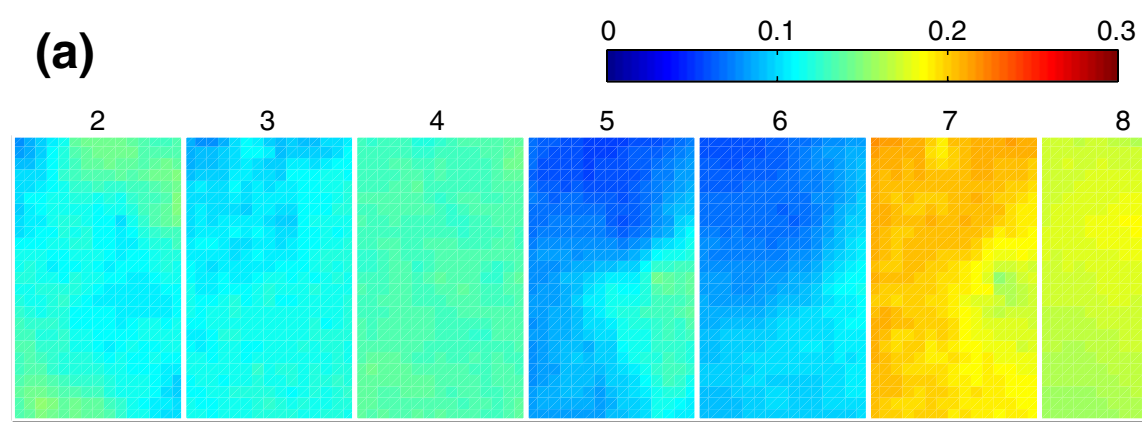

7

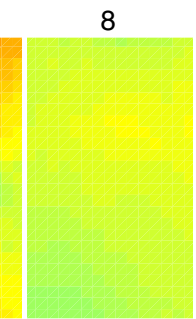

9
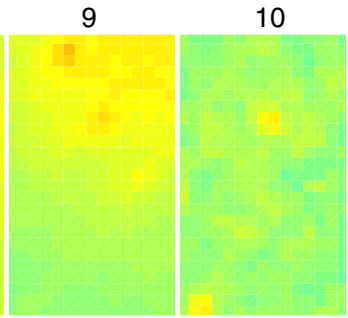

11

(b)
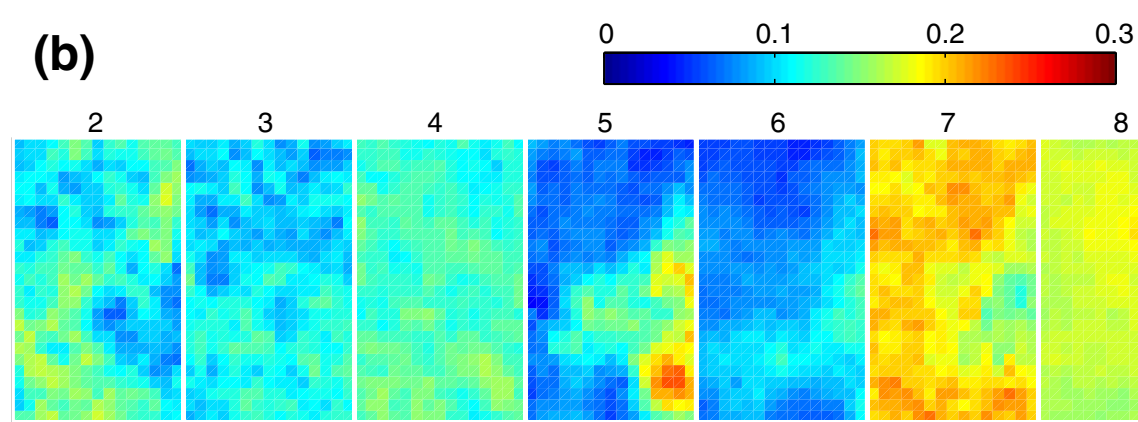

6

7

8

9

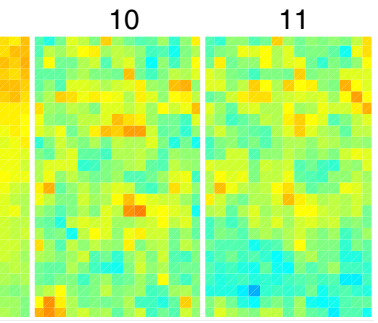

(c)
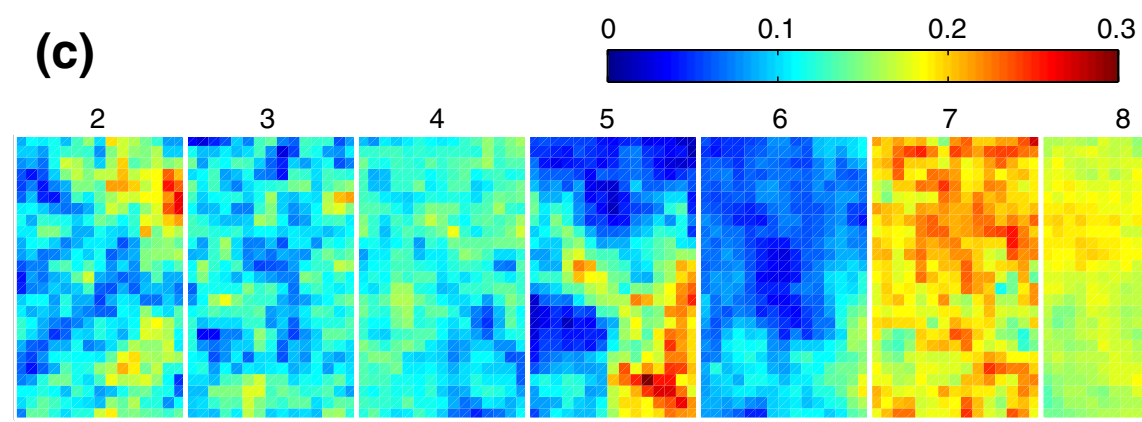

5
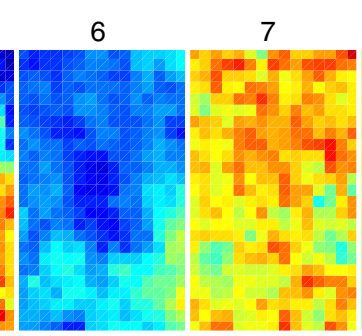

8

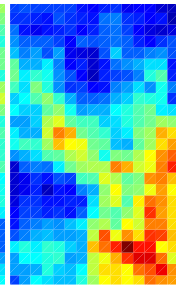

$0.1 \quad 0.2$
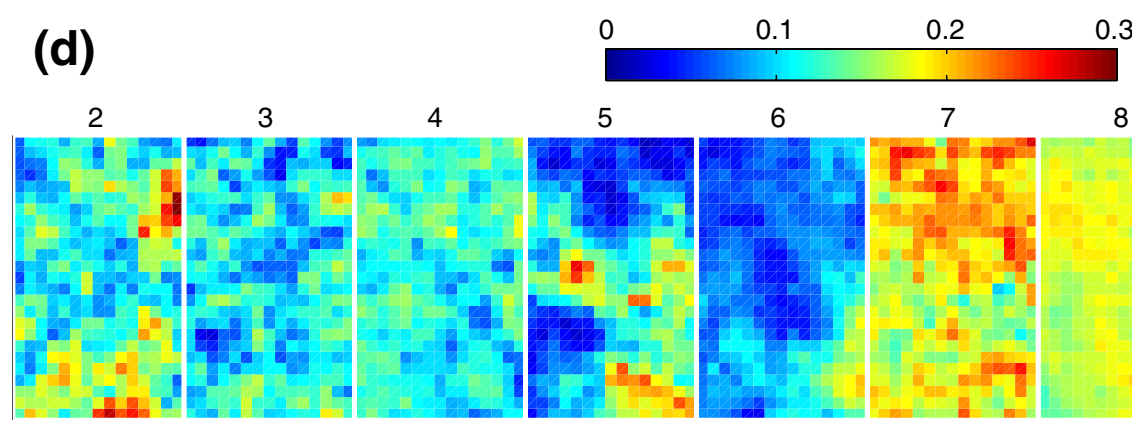

8

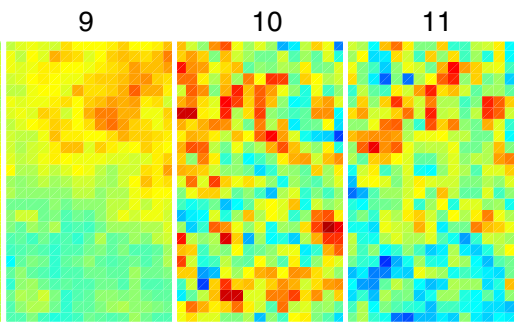

(e)

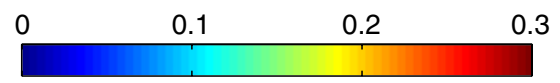

Fig. 14 a True porosity for layers 2 to 11 . b Initial ensemblemean porosity. c Ensemble-mean porosity resulting from an EnKF history match based on pressure and oil and water rates. d Ensemble-mean porosity conditioned to the base survey seismic. e Ensemble-mean porosity resulting from a combined history match to production and seismic data, starting from an ensemble conditioned to the base survey seismic, corresponding to Fig. 13(d) 
rather than the base survey. This turned out to result in a WCT profile with a slightly smaller RMSE value. The result of all the considered measures is that an improved forecast profile has resulted, when compared to the straightforward incorporation of seismic data, which leads to filter divergence. Further experiments were conducted in which additional iterations were attempted on the seismic update with the EnRML. These experiments involved rerunning all ensemble members from the initial time to the seismic update time and recomputing an ensemble-based downhill gradient direction with the EnRML scheme (i.e. towards a reduced cost function value). We applied this procedure both with 3D and with 4D seismic but could not further improve the match to the seismic data significantly beyond the first iteration. The estimated property fields for the experiments described above are shown in Fig. 14. The results are consistent with those of the WCT time series. While the broad distinction between reservoir zones of different porosity classes is already captured by the initial ensemble, as one would normally be able to do based on well log data and cores, details away from the wells are largely absent. Some of the main features of the true porosity are reproduced when production data is assimilated, but the amplitudes remain rather low compared with the true values. Much more detail, with more or less the correct amplitudes, is recovered by conditioning the ensemble to the base survey 3D seismic (Fig. 14d). From Fig. 14e we conclude that while we managed to produce a history match consistent with both production and seismic data, the property field did not improve much further by incorporating the timelapse seismic amplitudes.

\section{Summary and conclusions}

We have considered the ensemble-based history matching matching problem for combined production and seismic data, in which we have explored a forward modeling approach which does not require any seismic inversion in the traditional sense. Instead, seismic amplitudes are simulated using a 1D convolution model, which can subsequently be directly compared with the (processed) measured seismic data. An anticipated advantage is that the character of measurement errors is perhaps better understood in the amplitude domain, but this has not yet been tested with real data. The 3D model which was considered had a relatively simple configuration, but complex property distribution. An ensemble of relatively modest size (50 members) was used to set a somewhat realistic challenge. A sequential EnKF was observed to work quite well for history matching production data only. A study of ensemblebased covariances between model properties and the seismic amplitude response to dynamic changes in the reservoir suggested scope for improvement of the vertical property distribution. An iterative method was used successfully to condition the ensemble to the base survey seismic amplitude data, resulting in an improved property field and history match. Consistent with results reported elsewhere, however, the incorporation of relatively large numbers of high-accuracy seismic data during the history match period, was observed to lead to unsatisfactory results when no additional measures are taken. The use of a localization scheme helped to restore ensemble spread to some extent, but did not resolve the problem entirely. An additional improvement could be obtained by increasing the measurement error standard deviation whenever the data-model mismatch is too large. A simple adaptive inflation procedure further assisted in maintaining sufficient ensemble spread. Restarting the ensemble from the initial time after each update was found not to be necessary for maintaining spread or for stability of the runs with this model. Several alternative approaches were tested but did not lead to satisfactory results for this experimental case. While iterative conditioning to the initial 3D seismic proved successful, subsequent iteration on time-lapse seismic data was not as successful as anticipated. We conclude that while there are sufficient measures available to enable the incorporation of large numbers of data with the EnKF, the challenge remains to extract a maximum amount of information from the data. Further research into this issue should include the question whether it is always better to compute time-differences with respect to the most recent survey or to the base survey. We finally note here that while we updated saturation, in our simulator saturation is not a primary variable, and therefore a re-initialization step was required after each update.

The results suggest possible further lines of research. The observed problems with time-lapse seismic could possibly be resolved by applying a "global," instead of a "local" iteration. Using the concept of "asynchronous" data-assimilation [30] one could run the ensemble from the initial time all the way to the end of the history match period and perform a model update based on all simulated and measured data simultaneously, rather than sequentially. This would be similar to the approach taken in 4DVar, where data mismatches are collected over the entire model simulation period. Such an approach may require the use of inflation of the ensemble or of measurement errors, if the EnKF update scheme is used. An alternative is to use the EnRML, but this scheme, in particular the computation of the 
generalized inverse, Eq. 8, is found to be computationally costly when large numbers of data are used, since all dimensions involved are large. The numerical cost can possibly be reduced by switching from localization of the Kalman gain based on all measurements simultaneously, to assimilating uncorrelated batches of observations sequentially. Wang et al. [40] claimed that the EnRML scheme is not guaranteed to produce a downhill gradient direction due to all approximations involved, and that results may be rather sensitive to the choice of the step-size $\beta$. Further experimentation is needed to investigate this issue. There may be more to gain by further exploring more advanced adaptive inflation methods, as well as localization methods based on streamline simulation, both of which have not been considered in this study. Finally, Fahimuddin et al. [13] have compared the use of amplitude and impedance data and found the latter to produce somewhat better results. More research is needed, however, in order to fully understand the observed differences as well as potential benefits of specific types of seismic attributes.

Acknowledgements This work is supported by the Integrated Systems Approach Petroleum Production (ISAPP) consortium, and its partners TNO, Shell International Exploration and Production, and Delft University of Technology. The authors wish to thank the two anonymous reviewers for their constructive comments, which have helped to improve the manuscript considerably. The authors would also like to acknowledge the work of S. G. Douma and D. R. Brouwer (Shell) in developing the CLoReM closed-loop reservoir management software tool, and thank Shell International Exploration and Production for allowing the use of the MoReS reservoir simulator.

\section{References}

1. Anderson, J.L.: An adaptive covariance inflation error correction algorithm for ensemble filters. Tellus 59A, 210-224 (2007)

2. Burgers, G., van Leeuwen, P.J., Evensen, G.: Analysis scheme in the Ensemble Kalman Filter. Mon. Weather Rev. 126, 1719-1724 (1998)

3. Chen, Y., Oliver, D.S.: Cross-covariances and localization for EnKF in multiphase flow data assimilation. Comput. Geosci. (2009). doi:10.1007/210596-009-9174-6

4. Dadashpour, M., Landrø, M., Kleppe, J.: Nonlinear inversion for estimating reservoir parameters from time-lapse seismic data. J. Geophys. Eng. 5, 54-66 (2008)

5. Dadashpour, M., Echeverria-Ciaurri, D., Kleppe, J., Landrø, J.: Porosity and permeability estimation by integration of production and time-lapse near and far offset seismic data. J. Geophys. Eng. 6, 325-344 (2009)

6. Devegowda, D., Arroyo-Negrete, E., Datta-Gupta, A., Douma, S.G.: Efficient and robust reservoir model updating using Ensemble Kalman Filter with sensitivity-based covariance localization. SPE Reservoir Simulation Symposium, SPE, vol. 106144 (2007)
7. de Marseilly, G., Levendam, G., Boucher, M., Fasanino, G.: Interpretation of interference tests in a well field using geostatistical techniques to fit the permeability distributions in a reservoir model. In: Verly, G., David, M., Journel, A.G., Marechal, A. (eds.) Geostatistics for natural resource characterisation, Proc. NATO Advanced Study Inst. 831-849 (1984)

8. Dong, Y., Oliver, D.S.: Quantitative use of 4D seismic data for reservoir description. SPE J. SPE 84571, 91-99 (2005)

9. Dong, Y., Gu, Y., Oliver, D.S.: Sequential assimilation of 4D seismic data for reservoir description using the ensemble Kalman filter. J. Pet. Sci. Eng. 53, 83-99 (2006). doi:10.1016/j.petrol.2006.03.028

10. Evensen, G.: Sequential data assimilation with a nonlinear quasigeostrophic model using Monte Carlo methods to do forecast error statistics. J. Geophys. Res. 99(5), 10143-10162 (1994)

11. Evensen, G.: Using the EnKF for assisted history matching of a North Sea reservoir model. SPE Reservoir Simulation Symposium, SPE, vol. 106184 (2007)

12. Evensen, G.: Ensemble Kalman Filters for large geophysical applications. IEEE Control Systems Magazine (2009). doi:10.1109/MCS.2009.932222

13. Fahimuddin, A., Aanonsen, S.I., Skjervheim, J.-A.: Ensemble based 4D seismic history matching: integration of different levels and types of seismic data. SPE EUROPEC/EAGE Annual Conference and Exhibition, SPE, vol. 131453 (2010)

14. Furrer, R., Bengtsson, T.: Estimation of high-dimensional prior and posterior covariance matrices in Kalman filter variants. J. Multivar. Anal. 98, 227-255 (2007)

15. Gassmann, F.: Über die Elastizität poroser Medien. Vierteljahrsschr. Nat.forsch. Ges. Zür. 96, 251-262 (1951)

16. Gaspari, G., Cohn, S.E.: Construction of correlation functions in two and three dimensions. Q. J. R. Meteorol. Soc. 125(554), 723-757 (1999)

17. Gu, Y., Oliver, D.S.: An iterative Ensemble Kalman Filter for multiphase fluid flow data assimilation. SPE J. SPE 108438, 438-446 (2007)

18. Hammill, T.M., Whitaker, J.S., Snyder, C.: Distancedependent filtering of background error covariance estimates in an Ensemble Kalman Filter. Mon. Weather Rev. 129, 2776-2790 (2001)

19. Hashin, Z., Shtrikman, S.: A variational approach to the elastic behavior of multiphase material. J. Mech. Phys. Solids 11, 127-140 (1963)

20. Haverl, M.C., Skjervheim, J.-A., Landrø, M.: 4D seismic modeling integrated with the Ensemble Kalman Filter method for history matching of reservoir simulations models. European Conference on the Mathematics of Oil Recovery (2008)

21. Houtekamer, P., Mitchell, H.: A sequential Ensemble Kalman Filter for atmospheric data assimilation. Mon. Weather Rev. 129, 123-137 (2001)

22. Jazwinski, A.H.: Stochastic processes and filtering theory. Academic, San Diego (1970)

23. Jin, L., Sen, M.K., Stoffa, P.L., Seif, R.K.: Optimal model parameterization in stochastic inversion for reservoir properties using time-lapse seismic and production data. SEG/San Antionio 2007 Annual Meeting, pp 1805-1809 (2007)

24. Landrø, M.: Discrimination between pressure and fluid saturation from time-lapse seismic data. Geophysics 66(3), 836844 (2001)

25. Mindlin, R.D.: Compliance of elastic bodies in contact. J. Appl. Mech. 16, 259-268 (1949)

26. Naevdal, G., Johnsen, L.M., Aanonsen, S.I., Vefring, E.H.: Reservoir monitoring and continuous updating using 
Ensemble Kalma Filter. SPE Annual Technical Conference and Exhibition, SPE, vol. 84372 (2003)

27. Reynolds, A.C., Zafari, M., Li, G.: Iterative forms of the Ensemble Kalman Filter. In: Proceedings of the 10th European Conference on the Mathematics of Oil Recovery, Amsterdam, A030 (2006)

28. Sakov, P., Oke, P.R.: A deterministic formulation of the ensemble Kalman filter: an alternative to ensemble square-root filters. Tellus 60A, 361-371 (2008)

29. Sambridge, M.S.: Geopgysical inversion with a neighbourhood algorithm-I. Searching a parameter space. Geophys. J. Int. 138, 479-494 (1999)

30. Sakov, P., Evensen, G., Bertino, L.: Asynchronous data assimilation with the EnKF. Tellus 62A, 24-29 (2010). doi:10.1111/j.1600-0870.2009.00417.x

31. Sarma, P., Durlofsky, L.J., Aziz, K., Chen, W.H. : A new approach to automatic history matching using kernel PCA. SPE Reservoir Simulation Symposium, SPE, vol. 106176 (2007)

32. Skjervheim, J.-A., Evensen, G., Aanonsen, S.I., Ruud, B.O., Johansen, T.A.: Incorporating 4D seismic data in reservoir simulation models using ensemble Kalman filter. SPE Annual Technical Conference and Exhibition, SPE, vol. 95789 (2005)

33. Skjervheim, J.-A., Ruud, B.O.: Combined inversion of 4D seismic waveform data and production data using ensemble Kalman filter. SEG Expanded Abstracts 25, 1776 (2006). doi:10.1190/1.2369868
34. Stephen, K.D., Soldo, J., MacBeth, C., Christie, M.: Multiplemodel seismic and production history matching: a case study. SPE Europec/EAGE Annual Conference, SPE, vol. 94173 (2006)

35. Stephen, K.D., Shams, A., MacBeth, C.: Faster seismic history matching in a UKCS reservoir. SPE Europec/EAGE Annual Conference, SPE, vol. 107147 (2007)

36. Tippett, M., Anderson, J.L., Bishop, C.H., Hamill, T.M., Whitaker, J.S.: Ensemble square-root filters. Month. Weather Rev. 131, 1485-1490 (2003)

37. Trani, M., Arts, R., Leeuwenburgh, O., Brouwer, J., Douma, S.: The importance of localization for the assimilation of $4 \mathrm{D}$ seismic data using the EnKF. SEG Expanded Abstracts 28, 3835 (2009). doi:10.1190/1.3255667

38. Trani, M., Arts, R., Leeuwenburgh, O., Brouwer, J.: Estimation of changes in saturation and pressure from $4 \mathrm{D}$ seismic AVO and time-shift analysis. Geophysics (2010, accepted)

39. van Leeuwen, P.J.: Comment on data assimilation using an Ensemble Kalman Filter technique. Mon. Weather Rev. 127, 1374-1379 (1999)

40. Wang, Y., Li, G., Reynolds, A.C.: Estimation of depths of fluid contacts by history matching using iterative Ensemble Kalman Smoothers. SPE Reservoir Simulation Symposium, SPE, vol. 119056 (2009)

41. Wen, X.-H., Chen, W.H.: Real-time reservoir model updating using Ensemble Kalman Filter. SPE Reservoir Simulation Symposium, SPE, vol. 92991 (2005) 